\title{
Stille Cross-Couplings of Unactivated Secondary Alkyl Halides using Monoorganotin Reagents
}

\author{
David A. Powell, Toshihide Maki, and Gregory C. Fu* \\ Department of Chemistry, Massachusetts Institute of Technology, Cambridge, \\ Massachusetts 02139

\section{Supporting Information}

\section{General}

The following chemicals were purchased and used as received: $\mathrm{NiCl}_{2}$ (Alfa-Aesar or Strem), 2,2'-bipyridine (Alfa-Aesar or Aldrich), anhydrous KOt-Bu (Alfa-Aesar), anhydrous $t-\mathrm{BuOH}$ (Alfa-Aesar or Aldrich), anhydrous $i-\mathrm{BuOH}$ (Aldrich), $\mathrm{SnCl}_{4}$ (AlfaAesar), $\mathrm{PhSnCl}_{3}$ (Alfa-Aesar or Aldrich), 4-bromotoluene (Alfa-Aesar), 2-bromotoluene (Aldrich), 4-bromoanisole (Aldrich), 4-fluorobromobenzene (Alfa-Aesar), phenylacetylene (Alfa-Aesar), $\mathrm{Bu}_{3} \mathrm{SnH}$ (Aldrich), $\mathrm{AIBN}$ (Aldrich), $\mathrm{Mg}$ turnings (Fisher), 4-bromochlorobenzene (Aldrich), $\mathrm{Bu}_{3} \mathrm{SnCl}$ (Aldrich), $\mathrm{TBSCl}$ (Alfa-Aesar), imidazole (Avocado), $\mathrm{MeMgBr}$ (Aldrich), $\mathrm{PPh}_{3}$ (Alfa-Aesar), bromine (Alfa-Aesar), $\mathrm{N}$ bromosuccinimide (Aldrich), 2,3-dihydrofuran (Alfa-Aesar), TBAF (1.0 M in THF, Aldrich), 10-undecenal (Aldrich), cholesteryl chloroformate (Aldrich), pyrrole (Aldrich), 2,3-dihydrofuran (Aldrich), 1-bromo-6-chlorohexane (Alfa-Aesar), sodium iodide (AlfaAesar), 3-phenyl-2-propyn-1-ol (Alfa-Aesar), $\mathrm{PtO}_{2}$ (Aldrich), allyl alcohol (Aldrich), exo2-bromonorbornane (Aldrich), and 2-bromoadamantane (Aldrich).

The following chemicals were purchased and purified (by distillation and/or flash chromatography) prior to use: cyclohexyl bromide (Alfa-Aesar), cyclopentene (Fluka), 2-bromononane (Lancaster), cyclooctyl bromide (Alfa-Aesar), 1bromobenzocyclobutane (Aldrich), cyclopentyl bromide (Alfa-Aesar), and benzyl 4bromobutyl ether (Aldrich).

All experiments were conducted under an argon or nitrogen atmosphere in ovendried glassware with magnetic stirring, unless otherwise specified. 


\section{Preparation of Substrates}

\section{A. Preparation of Aryltrichlorotin Reagents}

General procedure. Magnesium turnings (1.1 equiv) and dry THF $(1 \mathrm{~mL} / \mathrm{mmol}$ of substrate) were added to a two-neck, round-bottom flask equipped with a reflux condenser. The aryl bromide (1.0 equiv) was added, and the reaction mixture was heated to $60^{\circ} \mathrm{C}$ for $3 \mathrm{~h}$ to ensure complete formation of the Grignard reagent. The solution was then cooled to room temperature, and $\mathrm{SnCl}_{4}(0.25$ equiv) was added dropwise over $30 \mathrm{~min}$. The resulting suspension was refluxed for $16 \mathrm{~h}$ and then cooled to room temperature. The reaction was quenched with saturated aqueous $\mathrm{NH}_{4} \mathrm{Cl}$ (added dropwise), and the reaction mixture was poured into a separatory funnel that contained aqueous $0.5 \mathrm{M} \mathrm{HCl}$. It was then extracted with EtOAc, and the combined organic layers were washed with brine, dried over $\mathrm{MgSO}_{4}$, filtered, and concentrated under reduced pressure to yield the tetraarylstannane.

The tetraarylstannane (1.0 equiv) was placed in a round-bottom flask equipped with a reflux condenser. $\mathrm{SnCl}_{4}$ (3.1 equiv) was added dropwise, and the resulting mixture was heated slowly to $150-200{ }^{\circ} \mathrm{C}$ for $4 \mathrm{~h}$. It was then cooled to room temperature, and the reflux condenser was exchanged for a short-path distillation head. The dark mixture was distilled under reduced pressure to afford the aryltrichlorotin reagent, which was stored under argon.

Trichloro-4-tolylstannane [51353-34-5]. This compound was prepared from 4bromotoluene ( $4.92 \mathrm{~mL}, 40.0 \mathrm{mmol})$. Purification by short-path distillation yielded 6.83 $\mathrm{g}$ (54\% yield for the two steps) of a colorless liquid (bp $83-85^{\circ} \mathrm{C}$ at 0.5 torr).

${ }^{1} \mathrm{H}$ NMR $\left(500 \mathrm{MHz}, \mathrm{CDCl}_{3}\right)$ d 7.60-7.53 $(2 \mathrm{H}, \mathrm{m})$, 7.47-7.37 $(2 \mathrm{H}, \mathrm{m}), 2.45(3 \mathrm{H}, \mathrm{s})$.

${ }^{13} \mathrm{CNMR}\left(125 \mathrm{MHz}, \mathrm{CDCl}_{3}\right) \delta$ 144.0, 133.8, 131.1, 124.7, 21.7.

${ }^{119} \mathrm{Sn}\left\{{ }^{1} \mathrm{H}\right\} \mathrm{NMR}\left(186 \mathrm{MHz}, \mathrm{CDCl}_{3}\right) \delta-55.9$.

Trichloro-2-tolylstannane [51729-82-9]. This compound was prepared from 2bromotoluene $(5.0 \mathrm{~mL}, 41.6 \mathrm{mmol})$. Purification by short-path distillation yielded $8.00 \mathrm{~g}$ (61\% yield for the two steps) of a colorless liquid (bp $95-98^{\circ} \mathrm{C}$ at 0.3 torr).

${ }^{1} \mathrm{H}$ NMR $\left(500 \mathrm{MHz}, \mathrm{CDCl}_{3}\right)$ \& 7.66-7.64 $(1 \mathrm{H}, \mathrm{m}), 7.57-7.54(1 \mathrm{H}, \mathrm{m}), 7.45-7.43(2 \mathrm{H}, \mathrm{m})$, $2.68(3 \mathrm{H}, \mathrm{s})$.

${ }^{13} \mathrm{CNMR}\left(125 \mathrm{MHz}, \mathrm{CDCl}_{3}\right) \delta$ 143.0, 136.8, 134.3, 133.3, 131.5, 127.1, 21.7.

${ }^{119} \mathrm{Sn}\left\{{ }^{1} \mathrm{H}\right\}$ NMR $\left(186 \mathrm{MHz}, \mathrm{CDCl}_{3}\right) \delta-60.4$.

Trichloro-(4-fluorophenyl)stannane [17236-60-1]. This compound was prepared from 4-fluorobromobenzene $(7.5 \mathrm{~mL}, 68.3 \mathrm{mmol})$. Purification by short-path distillation yielded $11.1 \mathrm{~g}$ (51\% yield for the two steps) of a light-yellow liquid (bp $105-108{ }^{\circ} \mathrm{C}$ at 0.7 torr).

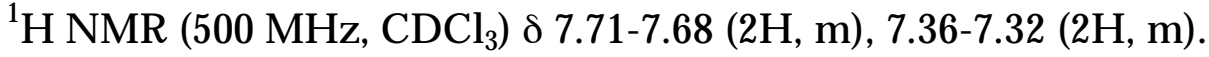


${ }^{13} \mathrm{CNMR}\left(125 \mathrm{MHz}, \mathrm{CDCl}_{3}\right) \delta 165.4\left(\mathrm{~d}, J_{\mathrm{C}-\mathrm{F}}=254 \mathrm{~Hz}\right), 136.2\left(\mathrm{~d}, J_{\mathrm{C}-\mathrm{F}}=8.0 \mathrm{~Hz}\right), 131.0(\mathrm{~d}$, $\left.J_{\mathrm{C}-\mathrm{F}}=4.0 \mathrm{~Hz}\right), 117.8\left(\mathrm{~d}, J_{\mathrm{C}-\mathrm{F}}=21.0 \mathrm{~Hz}\right)$.

${ }^{19} \mathrm{~F}\left(282 \mathrm{MHz}, \mathrm{CDCl}_{3}\right) \delta-103.4(\mathrm{~m})$.

${ }^{119} \mathrm{Sn}\left\{{ }^{1} \mathrm{H}\right\} \mathrm{NMR}\left(186 \mathrm{MHz}, \mathrm{CDCl}_{3}\right) \delta-57.4$.

Trichloro-(4-methoxyphenyl)stannane [51729-79-4]. This compound was prepared from 4-bromoanisole $(7.5 \mathrm{~mL}, 60.0 \mathrm{mmol})$. Purification by short-path distillation yielded $10.9 \mathrm{~g}$ (55\% yield for the two steps) of a light-yellow liquid (bp $115-118{ }^{\circ} \mathrm{C}$ at 0.4 torr).

${ }^{1} \mathrm{H} \mathrm{NMR}\left(500 \mathrm{MHz}, \mathrm{CDCl}_{3}\right)$ \& 7.59-7.55 (2H, m), 7.13-7.11 (2H, m), $3.88(3 \mathrm{H}, \mathrm{s})$.

${ }^{13} \mathrm{CNMR}\left(125 \mathrm{MHz}, \mathrm{CDCl}_{3}\right) \delta 163.2,135.4,126.3,115.9,55.5$.

${ }^{119} \mathrm{Sn}\left\{{ }^{1} \mathrm{H}\right\} \mathrm{NMR}\left(186 \mathrm{MHz}, \mathrm{CDCl}_{3}\right) \delta-54.7$. 


\section{B. Preparation of Aryl- and Alkenyltributyltin Reagents}

Tributyl-(4-chlorophenyl)stannane [17151-48-3]. A suspension of Mg turnings (0.99 $\mathrm{g}, 41.3 \mathrm{mmol})$ in THF $(60 \mathrm{~mL})$ was treated with 4-bromochlorobenzene $(7.50 \mathrm{~g}, 39.3$ mmol). The reaction mixture was heated to $50{ }^{\circ} \mathrm{C}$ for $3 \mathrm{~h}$, at which time no Mg could be seen. The reaction mixture was then cooled to room temperature, and $\mathrm{Bu}_{3} \mathrm{SnCl}(12.8$ $\mathrm{mL}, 47.2 \mathrm{mmol}$ ) was added. The reaction mixture was refluxed for $4 \mathrm{~h}$, and then cooled to room temperature and stirred overnight. The reaction was quenched by the dropwise addition of saturated aqueous $\mathrm{NH}_{4} \mathrm{Cl}$. The resulting mixture was poured into a separatory funnel that contained $\mathrm{H}_{2} \mathrm{O}(100 \mathrm{~mL})$ and then extracted with EtOAc $(3 \times 25$ $\mathrm{mL})$. The combined organic layers were washed with brine $(50 \mathrm{~mL})$, dried over $\mathrm{MgSO}_{4}$, filtered, and concentrated. Purification by column chromatography (eluant: $100 \%$ heptanes) furnished $10.5 \mathrm{~g}(67 \%)$ of the desired product as a colorless oil.

$\mathrm{R}_{f}=0.90$ (100\% hexanes).

${ }^{1} \mathrm{H}$ NMR $\left(500 \mathrm{MHz}, \mathrm{CDCl}_{3}\right)$ d 7.44-7.42 (2H, m), 7.35-7.33 (2H, m), 1.61-1.55 (6H, m), 1.41-1.34 (6H, m), 1.13-1.09 (6H, m), $0.94(9 \mathrm{H}, \mathrm{t}, J=7.5 \mathrm{~Hz})$.

${ }^{13} \mathrm{CNMR}\left(125 \mathrm{MHz}, \mathrm{CDCl}_{3}\right) \delta$ 140.0, 137.6, 134.3, 128.1, 29.0, 27.4, 13.7, 9.6.

(E)-1-Phenyl-(2-tributylstannyl)ethene [66680-88-4]. This compound was prepared from phenylacetylene $(1.65 \mathrm{~mL}, 15.0 \mathrm{mmol}), \mathrm{Bu}_{3} \mathrm{SnH}(4.00 \mathrm{~mL}, 15.0 \mathrm{mmol})$, and 2,2'azobisisobutyronitrile (AIBN; $100 \mathrm{mg}, 0.60 \mathrm{mmol}$ ) according to a literature procedure. ${ }^{1}$ The product was purified by column chromatography through a short plug of silica gel (eluant: hexanes), which furnished $4.25 \mathrm{~g}(74 \%)$ of a light-yellow oil $\left(>95 \%\right.$ pure by ${ }^{1} \mathrm{H}$ NMR).

$\mathrm{R}_{f}=0.90$ (100\% hexanes).

${ }^{1} \mathrm{H}$ NMR $\left(300 \mathrm{MHz}, \mathrm{CDCl}_{3}\right)$ \& 7.53-7.31 (5H, m), $6.99(2 \mathrm{H}, \mathrm{s}), 1.71-1.65(6 \mathrm{H}, \mathrm{m}), 1.50-$ $1.43(6 \mathrm{H}, \mathrm{m}), 1.12-0.99(15 \mathrm{H}, \mathrm{m})$.

${ }^{13} \mathrm{CNMR}\left(75 \mathrm{MHz}, \mathrm{CDCl}_{3}\right)$ \& 146.0, 138.8, 129.4, 128.4, 127.5, 126.0, 29.1, 27.3, 13.7, 9.6. 


\section{Preparation of Alkyl Halides}

Dodec-11-en-2-ol [21951-49-5]. A solution of 10-undecenal (5.00 mL, $24.1 \mathrm{mmol})$ in dry $\mathrm{Et}_{2} \mathrm{O}(25 \mathrm{~mL})$ was cooled to $0{ }^{\circ} \mathrm{C}$ in an ice bath. To the cooled solution was added $\mathrm{MeMgBr}\left(3.0 \mathrm{M}\right.$ in $\mathrm{Et}_{2} \mathrm{O} ; 16.0 \mathrm{~mL}, 48.2 \mathrm{mmol}$ ) over a 30-min period. The resulting solution was stirred at $0{ }^{\circ} \mathrm{C}$ for $2 \mathrm{~h}$, the ice bath was removed, and the mixture was stirred for $12 \mathrm{~h}$. The reaction was quenched by the dropwise addition of saturated aqueous $\mathrm{NH}_{4} \mathrm{Cl}(20 \mathrm{~mL})$ (caution: exothermic reaction), and the resulting suspension was poured into a separatory funnel that contained $1 \mathrm{M}$ aqueous $\mathrm{HCl}(50 \mathrm{~mL})$. The mixture was extracted with $\mathrm{Et}_{2} \mathrm{O}(3 \times 30 \mathrm{~mL})$, and the combined organic layers were washed with brine $(50 \mathrm{~mL})$, dried over $\mathrm{MgSO}_{4}$, and concentrated under reduced pressure. The crude reaction mixture was purified by column chromatography (eluant: 90:10 hexanes:EtOAc), which furnished $2.63 \mathrm{~g}(59 \%)$ of product as a colorless oil.

$\mathrm{R}_{f}=0.45$ (80:20 hexanes:EtOAc).

${ }^{1} \mathrm{H}$ NMR $\left(300 \mathrm{MHz}, \mathrm{CDCl}_{3}\right)$ 8 5.84-5.71 $(1 \mathrm{H}, \mathrm{m}), 4.98-4.87(2 \mathrm{H}, \mathrm{m}), 3.75-3.71(1 \mathrm{H}, \mathrm{m})$, $2.00(2 \mathrm{H}$, apparent quartet, $J=7.0 \mathrm{~Hz}), 1.89(1 \mathrm{H}, \mathrm{br} \mathrm{s}), 1.34-1.15(14 \mathrm{H}, \mathrm{m}), 1.13(3 \mathrm{H}, \mathrm{d}, J=$ $6.5 \mathrm{~Hz})$.

${ }^{13} \mathrm{C}$ NMR $\left(75 \mathrm{MHz}, \mathrm{CDCl}_{3}\right) \delta$ 139.1, 114.0, 67.9, 43.9, 33.7, 30.1, 29.6, 29.5, 29.4, 29.0, 29.1, 28.8.

11-Bromododec-1-ene. A solution of $\mathrm{PPh}_{3}(3.75 \mathrm{~g}$, $14.3 \mathrm{mmol})$ in $\mathrm{CH}_{2} \mathrm{Cl}_{2}(20 \mathrm{~mL})$ was cooled to $0{ }^{\circ} \mathrm{C}$ and treated with bromine $(730 \mu \mathrm{L})$. This mixture was stirred for $1 \mathrm{~h}$, and then a solution of dodec-11-en-2-ol $(2.50 \mathrm{~g}, 13.6 \mathrm{mmol})$ in $\mathrm{CH}_{2} \mathrm{Cl}_{2}(5 \mathrm{~mL})$ was added dropwise over $30 \mathrm{~min}$. The resulting solution was stirred at $0{ }^{\circ} \mathrm{C}$ for $1 \mathrm{~h}$, and then it was concentrated under reduced pressure to an oil, which was diluted with $\mathrm{Et}_{2} \mathrm{O}(50 \mathrm{~mL})$ and stirred vigorously to induce precipitation. The precipitate was removed by filtration through filter paper and washed with $\mathrm{Et}_{2} \mathrm{O}$. The resulting filtrate was poured into a separatory funnel containing a $5 \% \mathrm{w} / \mathrm{w}$ aqueous solution of $\mathrm{Na}_{2} \mathrm{SO}_{3}$. The organic layer was separated and washed with brine $(25 \mathrm{~mL})$, dried over $\mathrm{MgSO}_{4}$, filtered, and concentrated. The crude reaction product was purified by column chromatography (eluant: 100\% heptanes), which furnished $1.46 \mathrm{~g}(49 \%)$ of a colorless oil.

$\mathrm{R}_{f}=0.60$ (100\% hexanes).

${ }^{1} \mathrm{H}$ NMR $\left(300 \mathrm{MHz}, \mathrm{CDCl}_{3}\right) \delta 5.83(1 \mathrm{H}, \mathrm{ddt}, J=17.0,10.0,6.5 \mathrm{~Hz}), 4.98(1 \mathrm{H}$, apparent $\mathrm{dq}, J=17.0,1.5 \mathrm{~Hz}), 4.93(1 \mathrm{H}, \mathrm{ddt}, J=10.0,2.0,1.0 \mathrm{~Hz}), 4.14(1 \mathrm{H}, \mathrm{dqd}, J=8.0,7.0,5.5 \mathrm{~Hz})$, 2.08-2.01 $(2 \mathrm{H}, \mathrm{m}), 1.89-1.70(2 \mathrm{H}, \mathrm{m}), 1.71(3 \mathrm{H}, \mathrm{d}, J=6.5 \mathrm{~Hz}), 1.50-1.26(12 \mathrm{H}, \mathrm{m})$.

${ }^{13} \mathrm{C}$ NMR $\left(75 \mathrm{MHz}, \mathrm{CDCl}_{3}\right) \delta$ 139.1, 114.1, 51.9, 41.1, 33.8, 29.4, 29.3, 29.1, 28.9, 28.8, 27.7, 26.4.

IR (neat) 2926, 2855, 1641, 1464, 1378, 1226, $993,909 \mathrm{~cm}^{-1}$.

HRMS (EI) m/e [M- $\left.\mathrm{C}_{3} \mathrm{H}_{6}\right]^{+}$calcd for $\mathrm{C}_{9} \mathrm{H}_{17} \mathrm{Br}: 204.0508$, found 204.0519 .

trans-(2-Bromocyclohexyloxy)-tert-butyldimethylsilane. A solution of trans-2bromocyclohexanol $^{2}(2.20 \mathrm{~g}, 12.3 \mathrm{mmol}), \mathrm{TBSCl}$ ( $\left.2.83 \mathrm{~g}, 14.7 \mathrm{mmol}\right)$, and imidazole (1.00 $\mathrm{g}, 14.7 \mathrm{mmol})$ in dry DMF $(8 \mathrm{~mL})$ was stirred at room temperature for $1 \mathrm{~h}$. The mixture 
was then poured into $\mathrm{H}_{2} \mathrm{O}(50 \mathrm{~mL})$ and extracted with $\mathrm{Et}_{2} \mathrm{O}(3 \times 20 \mathrm{~mL})$. The combined organic layers were washed with brine $(20 \mathrm{~mL})$, dried over $\mathrm{MgSO}_{4}$, filtered, and concentrated. The reaction mixture was purified by column chromatography (eluant: $100 \%$ hexanes), which furnished $3.13 \mathrm{~g}$ (87\% yield) of the title compound as a clear oil.

$\mathrm{R}_{f}=0.35$ (100\% hexanes).

${ }^{1} \mathrm{H}$ NMR $\left(500 \mathrm{MHz}, \mathrm{CDCl}_{3}\right) \delta 3.90(1 \mathrm{H}, \mathrm{ddd}, J=9.5,7.5,4.0 \mathrm{~Hz}), 3.69(1 \mathrm{H}$, apparent $\mathrm{td}, J=7.5,4.0 \mathrm{~Hz}), 2.34-2.28(1 \mathrm{H}, \mathrm{m}), 2.05-2.00(1 \mathrm{H}, \mathrm{m}), 1.83-1.64(3 \mathrm{H}, \mathrm{m}), 1.41-1.28(3 \mathrm{H}$, m), $0.91(9 \mathrm{H}, \mathrm{s}), 0.13(3 \mathrm{H}, \mathrm{s}), 0.08(3 \mathrm{H}, \mathrm{s})$.

${ }^{13} \mathrm{C}$ NMR $\left(125 \mathrm{MHz}, \mathrm{CDCl}_{3}\right) \delta 74.6,58.1,34.7,34.0,25.8,25.0,23.0,18.1,-4.5,-4.6$. IR (neat) 2936, 1472, 1372, 1257, 1114, 837, 776, $669 \mathrm{~cm}^{-1}$. HRMS (EI) m/e [M- $\left.\mathrm{C}_{4} \mathrm{H}_{9}\right]^{+}$calcd for $\mathrm{C}_{8} \mathrm{H}_{16} \mathrm{BrOSi}$ : 235.0154, found 235.0171.

Cholesteryl iodide [2930-80-5]. This compound was prepared on a 5.0-mmol scale from cholesteryl chloroformate according to a literature procedure ${ }^{3}$. Recrystallization from acetone gave $3.83 \mathrm{~g}(70 \%)$ of a white crystalline solid.

Mp 106-107 ${ }^{\circ} \mathrm{C}$ (lit. mp 107-108 $\left.{ }^{\circ} \mathrm{C}\right) .^{3}$

${ }^{1} \mathrm{H}$ NMR $\left(300 \mathrm{MHz}, \mathrm{CDCl}_{3}\right) \delta 5.35-5.33(1 \mathrm{H}, \mathrm{m}), 4.05(1 \mathrm{H}$, apparent $\mathrm{tt}, J=12.0,4.5$ $\mathrm{Hz}), 2.98-2.88(1 \mathrm{H}, \mathrm{m}), 2.68(1 \mathrm{H}, \mathrm{ddd}, J=14.0,4.5,2.0 \mathrm{~Hz}), 2.32-2.18(2 \mathrm{H}, \mathrm{m}), 2.05-1.70$ $(4 \mathrm{H}, \mathrm{m}), 1.57-0.88(20 \mathrm{H}, \mathrm{m}), 1.05(3 \mathrm{H}, \mathrm{s}), 0.91(3 \mathrm{H}, \mathrm{d}, J=6.5 \mathrm{~Hz}), 0.88(3 \mathrm{H}, \mathrm{d}, J=6.5 \mathrm{~Hz})$, $0.86(3 \mathrm{H}, \mathrm{d}, J=6.5 \mathrm{~Hz}), 0.68(3 \mathrm{H}, \mathrm{s})$.

${ }^{13} \mathrm{C} \mathrm{NMR}\left(75 \mathrm{MHz}, \mathrm{CDCl}_{3}\right) \delta 142.7,121.7,56.6,56.1,50.3,46.4,42.3,41.9,39.6,39.5$, $36.6,36.4,36.1,35.8,31.7,31.6,30.5,28.2,28.0,24.2,23.8,22.8,22.6,20.8,19.2,18.7,11.8$. $[\alpha]_{\mathrm{D}}^{22}-13.4\left(\mathrm{c}=0.053 \mathrm{~g} / \mathrm{mL}, \mathrm{CHCl}_{3}\right)$.

1-(6-Chlorohexyl)-1H-pyrrole [144366-93-0]. 4 A mixture of pyrrole (1.25 mL, 18.0 $\mathrm{mmol})$ and finely crushed $\mathrm{KOH}(1.31 \mathrm{~g}, 23.4 \mathrm{mmol})$ in dry DMSO $(40 \mathrm{~mL})$ was sonicated for $20 \mathrm{~min}$ and then cooled to $0^{\circ} \mathrm{C}$. 1-Bromo-6-chlorohexane $(8.00 \mathrm{~mL}, 54.0 \mathrm{mmol})$ was added over $15 \mathrm{~min}$, and the resulting mixture was stirred at room temperature for $4 \mathrm{~h}$. The suspension was poured into a separatory funnel that contained $\mathrm{H}_{2} \mathrm{O}(150 \mathrm{~mL})$ and then extracted with EtOAc $(3 \times 50 \mathrm{~mL})$. The combined organic layers were washed with brine $(50 \mathrm{~mL})$, dried over $\mathrm{MgSO}_{4}$, filtered, and concentrated under reduced pressure. Purification by column chromatography (eluant: 100\% hexanes $\rightarrow$ 98:2 hexanes:EtOAc) furnished $2.69 \mathrm{~g}(81 \%)$ of the title compound as a colorless oil.

$\mathrm{R}_{f}=0.35$ (99:1 hexanes:EtOAc).

${ }^{1} \mathrm{H}$ NMR $\left(300 \mathrm{MHz}, \mathrm{CDCl}_{3}\right)$ d 6.76-6.73 (2H, m), 6.26-6.23 (2H, m), 3.99-3.94 (2H, m), 3.64-3.58 (2H, m), 1.92-1.80 (4H, m), 1.60-1.50 (2H, m), 1.45-1.35 (2H, m).

${ }^{13} \mathrm{C}$ NMR $\left(75 \mathrm{MHz} \mathrm{CDCl}_{3}\right) \delta 120.2,107.7,49.2,44.7,32.2,31.2,26.2,25.8$.

1-(6-Iodohexyl)-1H-pyrrole. A suspension of 1-(6-chlorohexyl)-1H-pyrrole (2.69 g, $14.5 \mathrm{mmol})$ and sodium iodide $(6.50 \mathrm{~g}, 43.6 \mathrm{mmol})$ in $\mathrm{MeCN}(80 \mathrm{~mL})$ was refluxed for 20 h. The cooled reaction mixture was poured into a separatory funnel that contained $\mathrm{H}_{2} \mathrm{O}$ $(100 \mathrm{~mL})$ and then extracted with $\mathrm{Et}_{2} \mathrm{O}(3 \times 30 \mathrm{~mL})$. The combined organic layers were washed with saturated aqueous $\mathrm{Na}_{2} \mathrm{SO}_{3}(50 \mathrm{~mL})$, brine $(50 \mathrm{~mL})$, dried over $\mathrm{MgSO}_{4}$, 
filtered, and concentrated under reduced pressure. Purification by column chromatography (eluant: 98:2 hexanes:EtOAc) furnished $2.13 \mathrm{~g}(53 \%)$ of the desired product as a slightly yellow oil.

$\mathrm{R}_{f}=0.35$ (99:1 hexanes:EtOAc).

${ }^{1} \mathrm{H}$ NMR $\left(300 \mathrm{MHz}, \mathrm{CDCl}_{3}\right) \delta 6.74(2 \mathrm{H}$, apparent $\mathrm{t}, J=2.0 \mathrm{~Hz}), 6.23(2 \mathrm{H}$, apparent $\mathrm{t}, J$ $=2.0 \mathrm{~Hz}), 3.96(2 \mathrm{H}, \mathrm{t}, J=7.0 \mathrm{~Hz}), 3.25(2 \mathrm{H}, \mathrm{t}, J=7.0 \mathrm{~Hz}), 1.94-1.81(4 \mathrm{H}, \mathrm{m}), 1.56-1.49(2 \mathrm{H}$, $\mathrm{m}), 1.46-1.34(2 \mathrm{H}, \mathrm{m})$.

${ }^{13} \mathrm{C}$ NMR $\left(75 \mathrm{MHz}, \mathrm{CDCl}_{3}\right) \delta 120.2,107.6,49.2,33.0,31.1,29.8,25.4,6.9$.

IR (neat) 2931, 2856, 1500, 1450, 1282, 1196, 1089, 722, $617 \mathrm{~cm}^{-1}$.

trans-1-Allyloxy-2-bromocyclopentane [174619-43-3]. Freshly distilled cyclopentene $(5.00 \mathrm{~mL}, 56.6 \mathrm{mmol}$ ) was added dropwise over a 20-min period to a suspension of $\mathrm{N}$-bromosuccinimide $(10.1 \mathrm{~g}, 56.6 \mathrm{mmol})$ in dry $\mathrm{CH}_{2} \mathrm{Cl}_{2}(100 \mathrm{~mL})$. The resulting yellow suspension was stirred at room temperature for $1 \mathrm{~h}$, and then allyl alcohol $(7.70 \mathrm{~mL}, 113.2 \mathrm{mmol})$ was added dropwise over $2 \mathrm{~h}$. The reaction mixture was stirred at room temperature for $4 \mathrm{~h}$, during which time it became colorless. The solvent was then removed, and the crude reaction mixture was poured into a separatory funnel that contained $\mathrm{H}_{2} \mathrm{O}(100 \mathrm{~mL})$. The mixture was extracted with $\mathrm{Et}_{2} \mathrm{O}(3 \times 50 \mathrm{~mL})$, and the combined organic layers were washed with brine $(50 \mathrm{~mL})$, dried over $\mathrm{MgSO}_{4}$, filtered, and concentrated under reduced pressure. Purification by column chromatography (eluant: $100 \%$ hexanes) furnished $0.82 \mathrm{~g}(7 \%)$ of the title compound as a clear oil.

$\mathrm{R}_{f}=0.25$ (100\% hexanes).

${ }^{1} \mathrm{H} \mathrm{NMR}\left(300 \mathrm{MHz}, \mathrm{CDCl}_{3}\right) \delta 5.88(1 \mathrm{H}, \mathrm{ddt}, J=17.0,10.5,5.5 \mathrm{~Hz}), 5.27(1 \mathrm{H}$, apparent $\mathrm{dq}, J=17.0,1.5 \mathrm{~Hz}), 5.17(1 \mathrm{H}$, apparent dq, $J=10.5,1.5 \mathrm{~Hz}), 4.26-4.24(1 \mathrm{H}, \mathrm{m}), 4.12-4.10$ $(1 \mathrm{H}, \mathrm{m}), 4.02(1 \mathrm{H}, \mathrm{ddt}, J=13.0,5.5,1.5 \mathrm{~Hz}), 3.99(1 \mathrm{H}, \mathrm{ddt}, J=13.0,5.5,1.5 \mathrm{~Hz}), 2.34-2.26$ $(1 \mathrm{H}, \mathrm{m}), 2.18-2.11(1 \mathrm{H}, \mathrm{m}), 2.03-1.97(1 \mathrm{H}, \mathrm{m}), 1.89-1.77(2 \mathrm{H}, \mathrm{m}), 1.68-1.62(1 \mathrm{H}, \mathrm{m})$.

${ }^{13} \mathrm{C}$ NMR $\left(75 \mathrm{MHz}, \mathrm{CDCl}_{3}\right) \delta$ 134.5, 116.9, 87.3, 70.3, 54.2, 34.7, 39.9, 21.7.

trans-2-Allyloxy-3-bromotetrahydrofuran [143037-46-1]. This compound was prepared on a $42.0 \mathrm{mmol}$ scale according to a literature procedure. ${ }^{5}$

$\mathrm{R}_{f}=0.40$ (95:5 hexanes: $\left.\mathrm{Et}_{2} \mathrm{O}\right)$.

${ }^{1} \mathrm{H}$ NMR (300 MHz, $\left.\mathrm{CDCl}_{3}\right) \delta 5.85(1 \mathrm{H}, \mathrm{dddd}, J=17.0,10.0,6.0,5.0 \mathrm{~Hz}), 5.27(1 \mathrm{H}$, ddt, $J=17.0,5.0,1.5 \mathrm{~Hz}), 5.23(1 \mathrm{H}, \mathrm{s}), 5.19-5.14(1 \mathrm{H}, \mathrm{ddt}, J=10.0,3.5,1.5 \mathrm{~Hz}), 4.23-3.91(5 \mathrm{H}$, $\mathrm{m})$, 2.69-2.57 $(1 \mathrm{H}, \mathrm{m}), 2.23-2.15(1 \mathrm{H}, \mathrm{m})$.

${ }^{13} \mathrm{C}$ NMR $\left(75 \mathrm{MHz}, \mathrm{CDCl}_{3}\right) \delta$ 133.9, 117.2, 107.8, 67.9, 66.6, 49.9, 33.8. 


\section{Stille Cross-Coupling Reactions of Alkyl Halides}

Preparation of a stock solution of $1.0 \mathrm{M} \mathrm{KO}$ - $\mathrm{Bu}$ in $t$-BuOH:i-BuOH (7:3). To a 200-mL round-bottom flask equipped with a reflux condenser was added $11.2 \mathrm{~g}(0.10$ $\mathrm{mol})$ of anhydrous $\mathrm{KO} t-\mathrm{Bu}$. Anhydrous $i-\mathrm{BuOH}(30 \mathrm{~mL})$ and anhydrous $t-\mathrm{BuOH}(50$ $\mathrm{mL}$ ) were added via cannula, and the resulting suspension was heated to $60^{\circ} \mathrm{C}$ for $3 \mathrm{~h}$ to ensure complete dissolution of the solids. The cooled, slightly yellow solution was transferred via cannula into an oven-dried bottle sealed with a septum. Anhydrous t$\mathrm{BuOH}$ was added until the total volume of the solution was $100 \mathrm{~mL}$.

Use of commercially available anhydrous $1.0 \mathrm{M} \mathrm{KO} t-\mathrm{Bu}$ in $t$-BuOH (Aldrich) and 1.0 $\mathrm{M} \mathrm{KO} i-\mathrm{Bu}$ in $i-\mathrm{BuOH}$ (Aldrich) in a 7:3 ratio leads to similar yields in the cross-coupling protocol.

Note: It is important that the $\mathrm{KOt}$ - $\mathrm{Bu}$ and the solvents are anhydrous and handled/stored under nitrogen or argon.

General procedure for Stille cross-couplings (Table 2, Table 3, and eq 4). Into a 20-mL sample vial was added $\mathrm{NiCl}_{2}(13 \mathrm{mg}, 0.10 \mathrm{mmol})$ and 2,2'-bipyridine $(23 \mathrm{mg}, 0.15$ $\mathrm{mmol})$; these compounds were weighed in air, and no special handling precautions were employed. The vial was sealed with a septum screw-cap and purged under a steady stream of argon (nitrogen may also be used) for $20 \mathrm{~min}$. A $1.0 \mathrm{M}$ stock solution of $\mathrm{KO} t$ - $\mathrm{Bu}$ in $t$ - $\mathrm{BuOH}: i-\mathrm{BuOH}(7: 3)(7.0 \mathrm{~mL}, 7.0 \mathrm{mmol}$ of $\mathrm{KO} t$-Bu) was added, followed by the aryltrichlorotin reagent $(1.2 \mathrm{mmol})$ and the alkyl halide $(1.0 \mathrm{mmol})$. The reaction mixture was heated in an oil bath to $60^{\circ} \mathrm{C}$ for $12 \mathrm{~h}$, with vigorous stirring under an argon atmosphere (the reaction mixture typically turns deep purple within $1 \mathrm{~h}$ of heating). Then, the cooled reaction mixture was poured into a separatory funnel that contained an aqueous $1 \mathrm{M} \mathrm{HCl}$ solution $(50 \mathrm{~mL})$, and the mixture was extracted with $\mathrm{Et}_{2} \mathrm{O}(3 \times 20 \mathrm{~mL})$. The combined organic layers were washed with brine $(40 \mathrm{~mL})$, dried over $\mathrm{MgSO}_{4}$, filtered, and concentrated under reduced pressure. The resulting crude reaction mixture was purified by column chromatography.

Note: The reaction is air- and moisture-sensitive. The KOt-Bu solution and the tin reagent should be handled/stored under nitrogen or argon.

(1-Methyloctyl)benzene (Table 2, entry 1) [51770-89-9]. Solvent used for chromatography: 100\% heptanes. Colorless oil. First run: $147 \mathrm{mg}(72 \%)$ of product. Second run: $146 \mathrm{mg}(72 \%)$ of product.

$\mathrm{R}_{f}=0.80$ (100\% hexanes).

${ }^{1} \mathrm{H}$ NMR $\left(300 \mathrm{MHz}, \mathrm{CDCl}_{3}\right) \delta$ 7.36-7.31 $(2 \mathrm{H}, \mathrm{m}), 7.26-7.20(3 \mathrm{H}, \mathrm{m}), 2.72(1 \mathrm{H}$, apparent sextet, $J=7.0 \mathrm{~Hz}), 1.64-1.59(2 \mathrm{H}, \mathrm{m}), 1.30-1.23(13 \mathrm{H}, \mathrm{m}), 0.93(3 \mathrm{H}, \mathrm{t}, J=6.5 \mathrm{~Hz})$.

${ }^{13} \mathrm{C}$ NMR $\left(75 \mathrm{MHz}, \mathrm{CDCl}_{3}\right) \delta 148.0,128.2,127.0,125.7,40.0,38.5,31.9,29.7,29.3,27.7$, 22.7, 22.3, 14.1. 
2-Phenyladamantane (Table 2, entry 2) [19066-24-1]. Solvent used for chromatography: 100\% heptanes. Colorless oil. First run: $160 \mathrm{mg}(75 \%)$ of product. Second run: $152 \mathrm{mg}(72 \%)$ of product.

$\mathrm{R}_{f}=0.75$ (100\% hexanes).

${ }^{1} \mathrm{H}$ NMR $\left(300 \mathrm{MHz}, \mathrm{CDCl}_{3}\right)$ d 7.46-7.36 (4H, m), 7.27-7.22 (1H, m), $3.09(1 \mathrm{H}, \mathrm{br} \mathrm{s}), 2.55$ (2H, br s), 2.11-1.85 (10H, m), 1.66-1.61 (2H, m).

${ }^{13} \mathrm{C}$ NMR $\left(75 \mathrm{MHz}, \mathrm{CDCl}_{3}\right) \delta 144.3,128.1,126.8,125.1,46.7,39.1,37.9,31.9,31.0,28.0$, 27.8.

7-p-Tolylbicyclo[4.2.0]octa-1,3,5-triene (Table 2, entry 3) [661476-70-6]. Solvent used for chromatography: 100\% heptanes. Colorless oil. First run: $159 \mathrm{mg}(82 \%)$ of product. Second run: $163 \mathrm{mg}(84 \%)$ of product.

$\mathrm{R}_{f}=0.35$ (100\% hexanes).

${ }^{1} \mathrm{H}$ NMR $\left(300 \mathrm{MHz}, \mathrm{CDCl}_{3}\right)$ 8 7.42-7.24 $(8 \mathrm{H}, \mathrm{m}), 4.90(1 \mathrm{H}, \mathrm{dd}, J=5.5,2.5 \mathrm{~Hz}), 3.84$ $(1 \mathrm{H}, \mathrm{dd}, J=14.0,5.5 \mathrm{~Hz}), 3.21(1 \mathrm{H}, \mathrm{dd}, J=14.0,2.5 \mathrm{~Hz}), 2.47(3 \mathrm{H}, \mathrm{s})$.

${ }^{13} \mathrm{C}$ NMR $\left(75 \mathrm{MHz}, \mathrm{CDCl}_{3}\right) \delta 147.9,144.1,139.8,135.9,129.1,127.5,127.0,126.8,123.2$, 122.6, 47.1, 40.0, 21.0.

1-Cyclohexyl-2-methylbenzene (Table 2, entry 4) [4501-35-3]. Solvent used for chromatography: 100\% heptanes. Colorless oil. First run: $84 \mathrm{mg}(48 \%)$ of product. Second run: $77 \mathrm{mg}(45 \%)$ of product.

$\mathrm{R}_{f}=0.60$ (100\% hexanes).

${ }^{1} \mathrm{H}$ NMR $\left(300 \mathrm{MHz}, \mathrm{CDCl}_{3}\right)$ \& 7.28-7.09 (4H, m), 2.80-2.70 (1H, m), $2.38(3 \mathrm{H}, \mathrm{s}), 1.91-$ $1.79(5 \mathrm{H}, \mathrm{m}), 1.54-1.38(5 \mathrm{H}, \mathrm{m})$.

${ }^{13} \mathrm{C}$ NMR $\left(75 \mathrm{MHz}, \mathrm{CDCl}_{3}\right) \delta$ 145.9, 135.1, 130.2, 126.1, 125.4, 125.3, 40.1, 33.6, 27.2, 26.3, 19.3.

(4-Fluorophenyl)cyclooctane (Table 2, entry 5). Solvent used for chromatography: $100 \%$ heptanes. Colorless oil. First run: $136 \mathrm{mg}(66 \%)$ of product. Second run: $140 \mathrm{mg}$ $(68 \%)$ of product.

$\mathrm{R}_{f}=0.20$ (100\% hexanes).

${ }^{1} \mathrm{H}$ NMR $\left(300 \mathrm{MHz}, \mathrm{CDCl}_{3}\right)$ d 7.19-7.14 (2H, m), 7.00-6.95 (2H, m), 2.82-2.75 (1H, m), $1.90-1.56(14 \mathrm{H}, \mathrm{m})$.

${ }^{13} \mathrm{C} \mathrm{NMR}\left(75 \mathrm{MHz}, \mathrm{CDCl}_{3}\right) \delta 161.3\left(\mathrm{~d}, J_{\mathrm{C}-\mathrm{F}}=243 \mathrm{~Hz}\right), 145.9\left(\mathrm{~d}, J_{\mathrm{C}-\mathrm{F}}=3.5 \mathrm{~Hz}\right), 128.1(\mathrm{~d}$, $\left.J_{\mathrm{C}-\mathrm{F}}=7.5 \mathrm{~Hz}\right), 114.8\left(\mathrm{~d}, J_{\mathrm{C}-\mathrm{F}}=21.0 \mathrm{~Hz}\right), 43.9,34.7,26.9,26.2,25.9$.

${ }^{19} \mathrm{~F}$ NMR $\left(282 \mathrm{MHz}, \mathrm{CDCl}_{3}\right) \delta-118.7(\mathrm{~m})$.

IR (neat) 2921, 1604, 1509, 1468, 1446, 1229, 1158, 838, $803 \mathrm{~cm}^{-1}$.

HRMS (EI) $m / e\left(\mathrm{M}^{+}\right)$calcd for $\mathrm{C}_{14} \mathrm{H}_{19} \mathrm{~F}: 206.1471$, found 206.1466 .

1-Methoxy-4-(1-methylundec-10-enyl)benzene (Table 2, entry 6). Solvent used for chromatography: 100\% hexanes. Light-yellow oil. First run: $192 \mathrm{mg}(70 \%)$ of product. Second run: $178 \mathrm{mg}(65 \%)$ of product.

$\mathrm{R}_{f}=0.20$ (100\% hexanes). 
${ }^{1} \mathrm{H}$ NMR $\left(300 \mathrm{MHz}, \mathrm{CDCl}_{3}\right)$ 8 7.27-7.12 (2H, m), 6.90-6.85 (2H, m), $5.86(1 \mathrm{H}, \mathrm{ddt}, J=$ 17.0, 10.0, $6.5 \mathrm{~Hz}), 5.07-4.95(2 \mathrm{H}, \mathrm{m}), 3.83(3 \mathrm{H}, \mathrm{s}), 2.73-2.61(1 \mathrm{H}$, apparent sextet, $J=7.0$ $\mathrm{Hz}), 2.11-2.03(2 \mathrm{H}, \mathrm{m}), 1.60-1.53(2 \mathrm{H}, \mathrm{m}), 1.43-1.27(12 \mathrm{H}, \mathrm{m}), 1.24(3 \mathrm{H}, \mathrm{d}, J=7.0 \mathrm{~Hz})$.

${ }^{13} \mathrm{C}$ NMR $\left(75 \mathrm{MHz}, \mathrm{CDCl}_{3}\right) \delta 157.6,140.0,139.2,127.7,114.1,113.6,55.1,39.0,38.6$, $33.8,29.7,29.5,29.4,29.1,28.9,27.7,22.5$.

IR (neat) 2926, 1612, 1513, 1247, 1178, 1040, 909, $828 \mathrm{~cm}^{-1}$.

HRMS (ESI) $m / e\left(\mathrm{M}+\mathrm{H}^{+}\right)$calcd for $\mathrm{C}_{19} \mathrm{H}_{31} \mathrm{O}: 275.2375$, found 275.2373 .

trans-tert-Butyl-[2-(4-fluorophenyl)cyclohexyloxy]dimethylsilane (Table 2, entry 7). GC-MS of the crude reaction mixture revealed a 96:4 mixture of stereoisomers, tentatively assigned as the trans and cis diastereomers. Solvent used for chromatography: 99:1 hexanes:EtOAc. Column chromatography was performed twice in order to remove impurities. A single diastereomer was isolated after column chromatography, and it was assigned as the trans isomer based on the magnitude of the $J_{\mathrm{H} 1-\mathrm{H} 2}$ coupling constant $(10 \mathrm{~Hz})$ in the ${ }^{1} \mathrm{H}$ NMR spectrum. First run: $151 \mathrm{mg}(49 \%)$ of product. Second run: $142 \mathrm{mg}(46 \%)$ of product. This compound was also characterized as the secondary alcohol.

$\mathrm{R}_{f}=0.25$ (100\% hexanes).

${ }^{1} \mathrm{H}$ NMR $\left(300 \mathrm{MHz}, \mathrm{CDCl}_{3}\right)$ \& 7.17-7.12 (2H, m), 6.98-6.93 (2H, m), 3.47 (1H, apparent $\mathrm{td}, J=10.0,4.5 \mathrm{~Hz}), 2.44(1 \mathrm{H}, \mathrm{ddd}, J=12.5,10.0,3.3 \mathrm{~Hz}), 2.00-1.97(1 \mathrm{H}, \mathrm{m}), 1.86-1.72(3 \mathrm{H}$, $\mathrm{m}), 1.60-1.46(1 \mathrm{H}, \mathrm{m}), 1.42-1.26(3 \mathrm{H}, \mathrm{m}), 0.68(9 \mathrm{H}, \mathrm{s}),-0.17(3 \mathrm{H}, \mathrm{s}),-0.50(3 \mathrm{H}, \mathrm{s})$.

${ }^{13} \mathrm{C} \mathrm{NMR}\left(75 \mathrm{MHz}, \mathrm{CDCl}_{3}\right) \delta 162.9\left(\mathrm{~d}, J_{\mathrm{C}-\mathrm{F}}=243 \mathrm{~Hz}\right), 140.7\left(\mathrm{~d}, J_{\mathrm{C}-\mathrm{F}}=3.0 \mathrm{~Hz}\right), 129.5(\mathrm{~d}$, $\left.J_{\mathrm{C}-\mathrm{F}}=7.5 \mathrm{~Hz}\right), 114.5\left(\mathrm{~d}, J_{\mathrm{C}-\mathrm{F}}=21.0 \mathrm{~Hz}\right), 76.0,52.2,36.6,32.8,26.0,25.6,25.2,17.8,-4.6,-5.6$.

${ }^{19} \mathrm{~F} \mathrm{NMR}\left(282 \mathrm{MHz}, \mathrm{CDCl}_{3}\right) \delta-118.3(\mathrm{~m})$.

IR (neat) 2931, 2857, 1606, 1583, 1511, 1250, 1228, 1098, 836, $775 \mathrm{~cm}^{-1}$.

trans-2-(4-Fluorophenyl)cyclohexanol. A solution of trans-tert-butyl-[2-(4fluorophenyl)cyclohexyloxy]dimethylsilane $(150 \mathrm{mg}, 0.49 \mathrm{mmol})$ in dry THF $(3 \mathrm{~mL})$ was treated with TBAF (1.0 M in THF; $1.46 \mathrm{~mL}, 1.46 \mathrm{mmol})$. The reaction was stirred at room temperature for $4 \mathrm{~h}$ and then concentrated. Purification by column chromatography (eluant: 90:10 hexanes:EtOAc) furnished $83 \mathrm{mg}(87 \%)$ of the secondary alcohol as a white solid.

Mp 70-71 ${ }^{\circ} \mathrm{C}$ (hexanes:EtOAc).

$\mathrm{R}_{f}=0.20$ (90:10 hexanes:EtOAc).

${ }^{1} \mathrm{H}$ NMR $\left(300 \mathrm{MHz}, \mathrm{CDCl}_{3}\right)$ \& 7.23-7.18 (2H, m), 7.04-6.99 (2H, m), 3.58 (1H, apparent $\mathrm{td}, J=10.0,4.1 \mathrm{~Hz}), 2.40(1 \mathrm{H}, \mathrm{ddd}, J=13.0,10.0,3.3 \mathrm{~Hz}), 2.11-2.07(1 \mathrm{H}, \mathrm{m}), 1.86-1.73(3 \mathrm{H}$, $\mathrm{m}), 1.50-1.34(4 \mathrm{H}, \mathrm{m})$.

${ }^{13} \mathrm{C} \mathrm{NMR}\left(75 \mathrm{MHz}, \mathrm{CDCl}_{3}\right) \delta 161.6\left(\mathrm{~d}, J_{\mathrm{C}-\mathrm{F}}=243 \mathrm{~Hz}\right), 139.0\left(\mathrm{~d}, J_{\mathrm{C}-\mathrm{F}}=3.0 \mathrm{~Hz}\right), 129.1\left(J_{\mathrm{C}-\mathrm{F}}\right.$ $=7.5 \mathrm{~Hz}), 115.3\left(J_{\mathrm{C}-\mathrm{F}}=21.0 \mathrm{~Hz}\right), 74.3,52.3,34.5,33.4,25.9,24.9$.

${ }^{19} \mathrm{~F}$ NMR $\left(282 \mathrm{MHz}, \mathrm{CDCl}_{3}\right) \delta-116.8(\mathrm{~m})$.

IR (KBr) 3333, 2936, 2855, 1605, 1510, 1228, 1060, $825 \mathrm{~cm}^{-1}$.

HRMS (ESI) $m / e\left(\mathrm{M}^{+}\right)$calcd for $\mathrm{C}_{12} \mathrm{H}_{15} \mathrm{FO}: 194.1107$, found 194.1099. 
3S-(4-Methoxylphenyl)cholesterol (Table 3, entry 1) [78406-16-3]. Solvent used for chromatography: 99:1 hexanes:EtOAc. White crystalline solid. Diastereomeric purity: >97:3. First run: $265 \mathrm{mg}$ (56\%) of product. Second run: $254 \mathrm{mg}$ (53\%) of product. The stereochemistry of the product was determined to be $3 S$ based on an X-ray crystal structure (X-ray quality crystals were obtained from hot acetone).

Mp 120-121 ${ }^{\circ} \mathrm{C}$ (hexanes:EtOAc).

$\mathrm{R}_{f}=0.20$ (99:1 hexanes:EtOAc).

${ }^{1} \mathrm{H}$ NMR $\left(500 \mathrm{MHz}, \mathrm{CDCl}_{3}\right)$ d 7.27-7.16 (2H, m), 6.87-6.85 (2H, m), $5.35(1 \mathrm{H}, \mathrm{dd}, J=$ 3.0, $2.0 \mathrm{~Hz}), 3.80(3 \mathrm{H}, \mathrm{s}), 2.48(1 \mathrm{H}$, apparent tt, $J=12.0,4.0 \mathrm{~Hz}), 2.43-2.38(1 \mathrm{H}, \mathrm{m}), 2.19-$ $2.16(1 \mathrm{H}, \mathrm{m}), 2.06-1.95(3 \mathrm{H}, \mathrm{m}), 1.87-1.83(1 \mathrm{H}, \mathrm{m}), 1.76-1.71(2 \mathrm{H}, \mathrm{m}), 1.64-1.46(6 \mathrm{H}, \mathrm{m})$, $1.42-0.99(14 \mathrm{H}, \mathrm{m}), 1.09(3 \mathrm{H}, \mathrm{s}), 0.94(3 \mathrm{H}, \mathrm{d}, J=6.5 \mathrm{~Hz}), 0.89(3 \mathrm{H}, \mathrm{d}, J=6.5 \mathrm{~Hz}), 0.87(3 \mathrm{H}$, $\mathrm{d}, J=6.5 \mathrm{~Hz}), 0.71(3 \mathrm{H}, \mathrm{s})$.

${ }^{13} \mathrm{C}$ NMR $\left(75 \mathrm{MHz}, \mathrm{CDCl}_{3}\right) \delta$ 157.7, 143.0, 139.3, 127.5, 120.0, 113.7, 56.8, 56.2, 55.2, $50.5,44.9,42.3,40.9,39.9,39.8,39.5,36.9,36.2,35.8,31.9,31.8,30.2,28.3,28.0,24.3,23.8$, $22.8,22.6,20.9,19.6,18.7,11.9$.

IR (KBr) 2931, 1612, 1511, 1461, 1365, 1240, 1040, 825, $801 \mathrm{~cm}^{-1}$.

HRMS (EI) $m / e\left(\mathrm{M}^{+}\right)$calcd for $\mathrm{C}_{34} \mathrm{H}_{52} \mathrm{O}: 476.4018$, found 476.4023 .

$[\alpha]_{\mathrm{D}}^{22}=-0.42\left(\mathrm{c}=0.034 \mathrm{~g} / \mathrm{mL}, \mathrm{CHCl}_{3}\right)$

(4-Benzyloxybutyl)benzene (Table 3, entry 2) [101747-16-4]. Solvent used for chromatography: 97:3 hexanes:EtOAc. Colorless oil. First run: $144 \mathrm{mg}(60 \%)$ of product. Second run: $146 \mathrm{mg}(61 \%)$ of product.

$\mathrm{R}_{f}=0.45$ (95:5 hexanes:EtOAc).

${ }^{1} \mathrm{H}$ NMR $\left(300 \mathrm{MHz}, \mathrm{CDCl}_{3}\right)$ d 7.41-7.31 (7H, m), 7.26-7.22 (3H, m), $4.55(2 \mathrm{H}, \mathrm{s}), 3.54$ $(2 \mathrm{H}, \mathrm{t}, J=6.0 \mathrm{~Hz}), 2.69(2 \mathrm{H}, \mathrm{t}, J=7.5 \mathrm{~Hz}), 1.81-1.72(4 \mathrm{H}, \mathrm{m})$.

${ }^{13} \mathrm{C}$ NMR $\left(75 \mathrm{MHz}_{2} \mathrm{CDCl}_{3}\right) \delta 142.4,138.5,128.4,128.3,128.2,127.6,127.4,125.6,72.8$, $70.1,35.7,29.4,28.0$.

1-(6-Phenylhexyl)-1H-pyrrole (Table 3, entry 3). Solvent used for chromatography: 99:1 hexanes: $\mathrm{Et}_{2} \mathrm{O}$. Colorless oil. First run: $143 \mathrm{mg}(63 \%)$ of product. Second run: $136 \mathrm{mg}(60 \%)$ of product

$\mathrm{R}_{f}=0.45$ (99:1 hexanes: $\left.\mathrm{Et}_{2} \mathrm{O}\right)$.

${ }^{1} \mathrm{H} \mathrm{NMR}\left(300 \mathrm{MHz}, \mathrm{CDCl}_{3}\right)$ \& 7.32-7.16 (5H, m), 6.66-6.65 (2H, m), 6.17-6.15 (2H, m), $3.87(2 \mathrm{H}, \mathrm{t}, J=7.0 \mathrm{~Hz}), 2.61(2 \mathrm{H}, \mathrm{t}, J=7.5 \mathrm{~Hz}), 1.82-1.73(2 \mathrm{H}, \mathrm{m}), 1.68-1.54(2 \mathrm{H}, \mathrm{m}), 1.39-$ $1.28(4 \mathrm{H}, \mathrm{m})$.

${ }^{13} \mathrm{C}$ NMR $\left(75 \mathrm{MHz}, \mathrm{CDCl}_{3}\right) \delta 142.5,128.3,128.2,125.6,120.4,107.7,49.5,35.8,31.5$, $31.3,28.8,26.6$.

IR (neat) 2931, 2856, 1499, 1453, 1281, 1089, 1062, 722, $618 \mathrm{~cm}^{-1}$.

HRMS (EI) $m / \mathrm{e}\left(\mathrm{M}^{+}\right)$calcd for $\mathrm{C}_{16} \mathrm{H}_{21} \mathrm{~N}$ : 227.1674, found: 227.1674.

General procedure for eq 2 and eq 3. In a glovebox, the tin reagent $(0.60 \mathrm{mmol})$ and $\mathrm{SnCl}_{4}(70 \mu \mathrm{L}, 0.60 \mathrm{mmol})$ were added to a $20-\mathrm{mL}$ sample vial, and the resulting solution was stirred for $1 \mathrm{~h}$ (vinylstannane) or $6 \mathrm{~h}$ (arylstannane). To the neat reaction 
mixture was added $\mathrm{NiCl}_{2}$ (7.0 mg, $\left.0.050 \mathrm{mmol}\right), 2$,2'-bipyridine (12 mg, $0.075 \mathrm{mmol}$ ), and a stock solution of $\mathrm{KO} t-\mathrm{Bu}$ in $t$-BuOH:i-BuOH $(7: 3)(1.0 \mathrm{M} ; 3.5 \mathrm{~mL}, 3.5 \mathrm{mmol}$ of $\mathrm{KOt}$ $\mathrm{Bu}$ ). The suspension was stirred at room temperature for $10 \mathrm{~min}$, and then the alkyl halide $(0.5 \mathrm{mmol})$ was added. The vial was sealed with a teflon-coated screw cap, taped, and heated to $60^{\circ} \mathrm{C}$ for $20 \mathrm{~h}$. Then, the cooled reaction mixture was diluted with a saturated aqueous solution of KF (15 mL) and filtered through a pad of celite on a sintered glass funnel, washing with $\mathrm{Et}_{2} \mathrm{O}$. The filtrate was poured into a separatory funnel that contained an aqueous $1 \mathrm{M} \mathrm{HCl}$ solution $(50 \mathrm{~mL})$, and the mixture was extracted with $\mathrm{Et}_{2} \mathrm{O}(3 \times 20 \mathrm{~mL})$. The combined organic layers were washed with brine $(40 \mathrm{~mL})$, dried over $\mathrm{MgSO}_{4}$, filtered, and concentrated under reduced pressure. The resulting crude reaction mixture was purified by column chromatography.

1-Chloro-4-cyclopentylbenzene [91335-74-9]. Solvent used for chromatography: 100\% heptanes. Colorless oil. First run: $60 \mathrm{mg}$ (66\%) of product. Second run: $59 \mathrm{mg}$ $(66 \%)$ of product.

$\mathrm{R}_{f}=0.75$ (100\% hexanes).

${ }^{1} \mathrm{H}$ NMR $\left(300 \mathrm{MHz}, \mathrm{CDCl}_{3}\right)$ \& 7.28-7.25 (2H, m), 7.21-7.17 (2H, m), $2.98(1 \mathrm{H}, \mathrm{tt}, J=9.5$, $7.5 \mathrm{~Hz}), 2.13-2.03(2 \mathrm{H}, \mathrm{m}), 1.88-1.65(4 \mathrm{H}, \mathrm{m}), 1.63-1.50(2 \mathrm{H}, \mathrm{m})$.

${ }^{13} \mathrm{C}$ NMR $\left(75 \mathrm{MHz}, \mathrm{CDCl}_{3}\right) \delta 144.9,131.2,128.4,128.2,45.3,34.6,25.4$.

HRMS (EI) $m / e\left(\mathrm{M}^{+}\right)$calcd for $\mathrm{C}_{11} \mathrm{H}_{13} \mathrm{Cl}$ : 180.0706, found 180.0707 .

exo-(E)-2-Styrylbicyclo[2.2.1]heptane [76217-04-4]. Solvent used for chromatography: $100 \%$ heptanes. Colorless oil. First run: $60 \mathrm{mg}(61 \%)$ of product. Second run: $61 \mathrm{mg}(62 \%)$ of product. ${ }^{1} \mathrm{H}$ NMR analysis revealed that the $(E)$-alkene was formed in a 98:2 ratio. The stereochemistry at the 2 position was deduced through conversion to the known bicyclo[2.2.1] heptane-2-carboxylic acid 6 through oxidative cleavage of the alkene with $\mathrm{KMnO}_{4}$ fused on silica gel. ${ }^{7}$ A sample of commercially available bicyclo[2.2.1] heptane-2-carboxylic acid (endo:exo $=67: 33$, as determined by ${ }^{1} \mathrm{H}$ NMR analysis) did not isomerize when subjected to the oxidative conditions described above.

$\mathrm{R}_{f}=0.65$ (100\% hexanes).

${ }^{1} \mathrm{H}$ NMR $\left(300 \mathrm{MHz}, \mathrm{CDCl}_{3}\right)$ d 7.45-7.34 $(4 \mathrm{H}, \mathrm{m}), 7.30-7.24(1 \mathrm{H}, \mathrm{m}), 6.40(1 \mathrm{H}, \mathrm{d}, J=16.0$ $\mathrm{Hz}), 6.22(1 \mathrm{H}, \mathrm{dd}, J=16.0,8.0 \mathrm{~Hz}), 2.40-2.33(2 \mathrm{H}, \mathrm{m}), 2.25-2.22(1 \mathrm{H}, \mathrm{m}), 1.70-1.24(8 \mathrm{H}$, $\mathrm{m})$.

${ }^{13} \mathrm{C}$ NMR $\left(75 \mathrm{MHz}, \mathrm{CDCl}_{3}\right) \delta 137.9,136.3,128.4,127.2,126.6,125.9,45.4,42.7,37.9$, $36.6,35.8,29.7,29.0$.

IR (neat) 3025, 2959, 2869, 1598, 1497, 1449, 1310, 963, 743, $692 \mathrm{~cm}^{-1}$.

HRMS (EI) $m / e\left(\mathbf{M}^{+}\right)$calcd for $\mathrm{C}_{15} \mathrm{H}_{18}: 198.1409$, found: 198.1403 .

3-Benzylhexahydrocyclopenta[b]furan (3; eq 4). GC analysis of the crude reaction mixture revealed a 68:32 mixture of diastereomers. These diastereomers were difficult to separate by column chromatography and were isolated as a mixture. Solvent used for chromatography: 95:5 hexanes: $\mathrm{Et}_{2} \mathrm{O}$. Clear oil. First run: $131 \mathrm{mg}(65 \%)$ of product. 
Second run: $137 \mathrm{mg}(68 \%)$ of product. The diastereomers were separated by preparative HPLC (Alltech Econosphere $10 \mu$ silica gel column, $250 \mathrm{~mm} \times 22 \mathrm{~mm}$, eluted with 95:5 hexanes: $\mathrm{Et}_{2} \mathrm{O}$ at a flow rate of $10 \mathrm{~mL} / \mathrm{min}$, observing at $210 \mathrm{~nm}$ ).

$\left(3 R^{*}, 3 \mathrm{a} S^{*}, 6 \mathrm{a} R^{*}\right)$-3-Benzylhexahydrocyclopenta[ $\left.b\right]$ furan (major diastereomer).

$\mathrm{R}_{f}=0.15$ (95:5 hexanes: $\mathrm{Et}_{2} \mathrm{O}$ ).

${ }^{1} \mathrm{H}$ NMR $\left(500 \mathrm{MHz}, \mathrm{CDCl}_{3}\right)$ \& 7.31-7.27 (2H, m), 7.22-7.19 (3H, m), 4.52 (1H, apparent $\mathrm{td}, J=6.0,2.0 \mathrm{~Hz}), 3.82(1 \mathrm{H}, \mathrm{dd}, J=8.5,7.0 \mathrm{~Hz}), 3.46(1 \mathrm{H}, \mathrm{dd}, J=10.0,8.5 \mathrm{~Hz}), 2.77-2.72$ $(1 \mathrm{H}, \mathrm{m}), 2.68-2.62(2 \mathrm{H}, \mathrm{m}), 2.54-2.49(1 \mathrm{H}, \mathrm{m}), 1.83-1.51(6 \mathrm{H}, \mathrm{m})$.

${ }^{13} \mathrm{C}$ NMR $\left(75 \mathrm{MHz}, \mathrm{CDCl}_{3}\right) \delta$ 141.0, 128.5, 128.4, 125.9, 86.1, 71.9, 46.4, 44.9, 34.6, 34.1, 26.0, 25.4.

IR (neat) $3027,2954,1603,1496,1453,1150,1041,752,700 \mathrm{~cm}^{-1}$.

HRMS (EI) $m / \mathrm{e}\left(\mathrm{M}^{+}\right)$calcd for $\mathrm{C}_{14} \mathrm{H}_{18} \mathrm{O}: 202.1358$, found: 202.1348 .

$\left(3 R^{*}, 3 a R^{*}, 6 a R^{*}\right)-3-B e n z y l h e x a h y d r o c y c l o p e n t a[b]$ furan (minor diastereomer). $\mathrm{R}_{f}=0.15$ (95:5 hexanes: $\left.\mathrm{Et}_{2} \mathrm{O}\right)$.

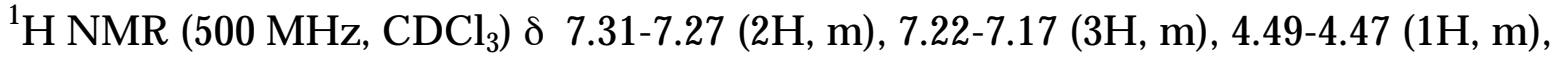
$3.85(1 \mathrm{H}, \mathrm{dd}, J=8.5,6.5 \mathrm{~Hz}), 3.33(1 \mathrm{H}$, apparent $\mathrm{t}, J=8.5 \mathrm{~Hz}), 2.75(1 \mathrm{H}, \mathrm{dd}, J=13.5,7.0$ $\mathrm{Hz}), 2.67(1 \mathrm{H}, \mathrm{dd}, J=13.5,8.0 \mathrm{~Hz}), 2.34-2.30(1 \mathrm{H}, \mathrm{m}), 2.10-2.03(1 \mathrm{H}, \mathrm{m}), 1.81-1.69(1 \mathrm{H}$, $\mathrm{m}), 1.59-1.48(4 \mathrm{H}, \mathrm{m}), 1.35-1.33(1 \mathrm{H}, \mathrm{m})$.

${ }^{13} \mathrm{C}$ NMR $\left(75 \mathrm{MHz}, \mathrm{CDCl}_{3}\right) \delta$ 140.7, 128.6, 128.4, 126.0, 85.1, 73.1, 49.4, 49.1, 39.0, 34.1, 32.2, 23.9.

IR (neat) 3026, 2932, 1604, 1496, 1453, 1205, 1049, 745, $700 \mathrm{~cm}^{-1}$.

HRMS (EI) m/e (M ${ }^{+}$) calcd for $\mathrm{C}_{14} \mathrm{H}_{18} \mathrm{O}: 202.1358$, found: 202.1352 .

Determination of the relative stereochemistry via independent synthesis.
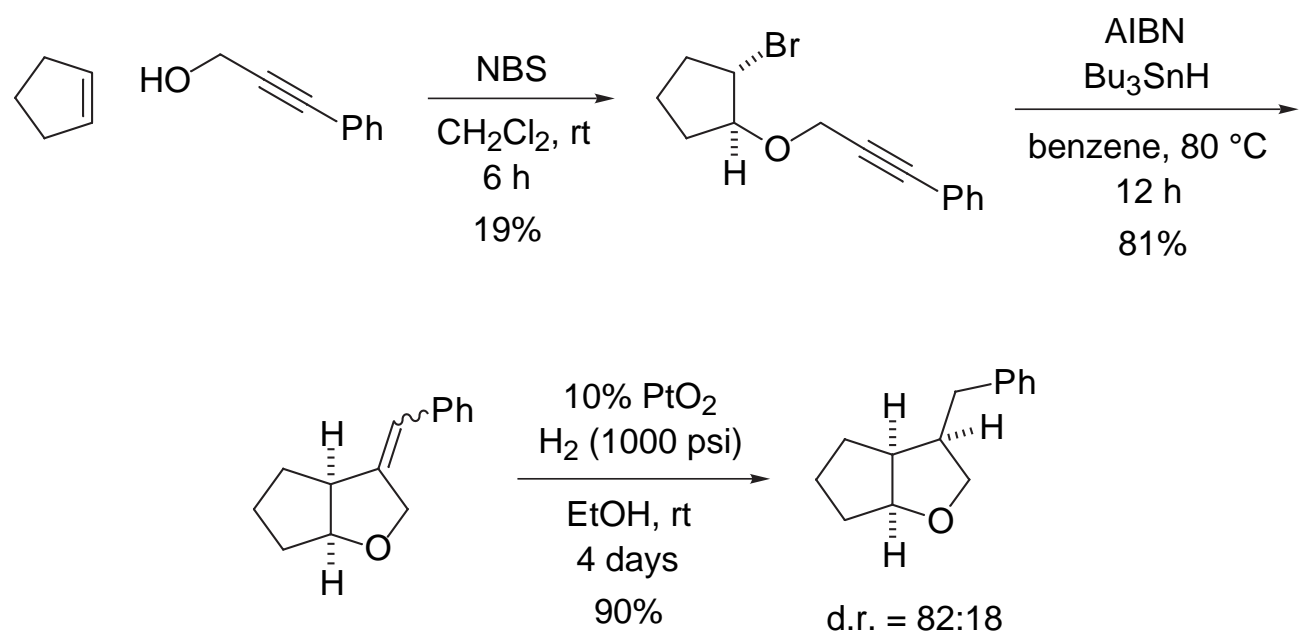

[3-(2-Bromocyclopentyloxy)prop-1-ynyl]benzene. $N$-Bromosuccinimide was added to a $250-\mathrm{mL}$ round-bottom flask $(10.1 \mathrm{~g}, 56.8 \mathrm{mmol})$ that contained a solution of freshly distilled cyclopentene $(5.02 \mathrm{~mL}, 56.8 \mathrm{mmol})$ in $\mathrm{CH}_{2} \mathrm{Cl}_{2}(100 \mathrm{~mL})$. 3-Phenyl-2-propyn-1-ol $(4.64 \mathrm{~mL}, 37.9 \mathrm{mmol})$ was added dropwise via syringe pump to this suspension over a 4-h period. The reaction mixture was stirred at room temperature for an additional 12 $h$, and then the opaque yellow suspension was concentrated under reduced pressure. 
The residue was taken up in $\mathrm{Et}_{2} \mathrm{O}(50 \mathrm{~mL})$ and poured into a $250-\mathrm{mL}$ separatory funnel that contained $\mathrm{H}_{2} \mathrm{O}(100 \mathrm{~mL})$. The aqueous layer was extracted with $\mathrm{Et}_{2} \mathrm{O}(3 \times 30 \mathrm{~mL})$, and the combined organic layers were washed with brine $(50 \mathrm{~mL})$, dried over $\mathrm{MgSO}_{4}$, filtered, and concentrated under reduced pressure. Purification by column chromatography (eluant: 99:1 hexanes: $\left.\mathrm{Et}_{2} \mathrm{O}\right)$ furnished $1.96 \mathrm{~g}(19 \%)$ of the product as a light-yellow oil.

$\mathrm{R}_{f}=0.15$ (hexanes).

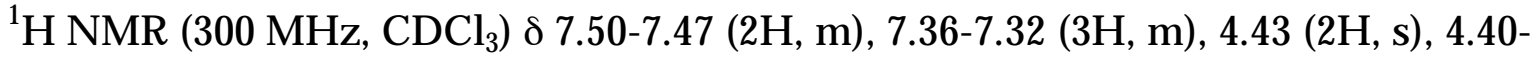
$4.34(2 \mathrm{H}, \mathrm{m}), 2.41-2.20(2 \mathrm{H}, \mathrm{m}), 2.10-2.00(1 \mathrm{H}, \mathrm{m}), 1.92-1.71(3 \mathrm{H}, \mathrm{m})$.

${ }^{13} \mathrm{C} \mathrm{NMR}\left(75 \mathrm{MHz}, \mathrm{CDCl}_{3}\right) \delta 131.7,128.5,128.2,122.4,87.4,86.2,84.9,57.6,54.0,34.7$, $29.9,21.7$.

IR (neat) 2970, 2190, 1662, 1490, 1351, 1078, 757, $691 \mathrm{~cm}^{-1}$.

HRMS (ESI) m/e (M+Na ${ }^{+}$) calcd for $\mathrm{C}_{14} \mathrm{H}_{15} \mathrm{BrONa}$ : 301.0204, found: 301.0208 .

3-Benzylhexahydrocyclopenta[b]furan (3). A solution of [3-(2bromocyclopentyloxy)prop-1-ynyl]benzene (1.00 g, $3.58 \mathrm{mmol}), 2$,2'azobisisobutyronitrile (AIBN; $20 \mathrm{mg}, 0.18 \mathrm{mmol}$ ), and $\mathrm{Bu}_{3} \mathrm{SnH}(1.05 \mathrm{~mL}, 3.94 \mathrm{mmol}$ ) in benzene $(36 \mathrm{~mL})$ was heated to reflux for $12 \mathrm{~h}$. The cooled reaction mixture was concentrated under reduced pressure and purified by column chromatography (eluant: 99:1 hexanes: $\left.\mathrm{Et}_{2} \mathrm{O}\right)$, which furnished $582 \mathrm{mg}(81 \%)$ of a clear oil that was used directly in the next step.

A suspension of the above compound $(100 \mathrm{mg}, 0.50 \mathrm{mmol})$ and $\mathrm{PtO}_{2}(11 \mathrm{mg}, 0.050$ $\mathrm{mmol})$ in $\mathrm{EtOH}(4 \mathrm{~mL})$ was stirred at room temperature in a high-pressure hydrogenation apparatus under a pressure of 1000 psi of hydrogen for 4 days. The reaction mixture was then filtered through a pad of celite on a sintered glass funnel (Et ${ }_{2} \mathrm{O}$ washings), and the filtrate was concentrated under reduced pressure. GC analysis revealed an 82:18 ratio of diastereomers. The reaction mixture was purified by column chromatography (eluant: 90:10 hexanes: $\left.\mathrm{Et}_{2} \mathrm{O}\right)$, which furnished $90 \mathrm{mg}(90 \%)$ of a clear oil. The major diastereomer is identical (GC and ${ }^{1} \mathrm{H}$ NMR) with the major diastereomer 3 from the cyclization/cross-coupling reaction illustrated in eq 4 . Based on related hydrogenations of methylenehexahydrofuro[2,3-b]furan ${ }^{8}$ and the expected delivery of hydrogen from the less hindered convex face, we have assigned the major diastereomer as the $3 R^{*}, 3 \mathrm{a} S^{*}, 6 \mathrm{a} R^{*}(e n d o)$ isomer.

$\left(3 R^{*}, 3 \mathrm{a} S^{*}, 6 \mathrm{a} R^{*}\right)-3-$ Benzylhexahydrofuro[2,3-b]furan (4; eq 4). GC analysis of the crude reaction mixture revealed a 96:4 mixture of diastereomers. Solvent used for chromatography: 90:10 hexanes: $\mathrm{Et}_{2} \mathrm{O}$. Slightly yellow oil. First run: $116 \mathrm{mg}(57 \%)$ of product. Second run: $117 \mathrm{mg}(57 \%)$ of product.

$\mathrm{R}_{f}=0.07$ (90:10 hexanes: $\left.\mathrm{Et}_{2} \mathrm{O}\right)$.

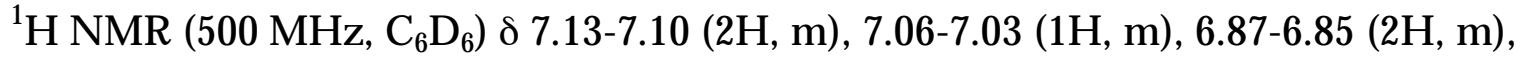
$5.70(1 \mathrm{H}, \mathrm{d}, J=5.0 \mathrm{~Hz}), 3.68-3.64(2 \mathrm{H}, \mathrm{m}), 3.57-3.53(1 \mathrm{H}, \mathrm{m}), 3.33-3.27(1 \mathrm{H}, \mathrm{m}), 2.22-2.12$ $(2 \mathrm{H}, \mathrm{m}), 2.19(2 \mathrm{H}, \mathrm{d}, J=2.5 \mathrm{~Hz}), 1.42(1 \mathrm{H}, \mathrm{ddt}, J=12.5,7.0,5.0 \mathrm{~Hz}), 1.24(1 \mathrm{H}, \mathrm{ddt}, J=$ $12.5,10.0,7.5 \mathrm{~Hz}$ ). 
${ }^{13} \mathrm{C} \mathrm{NMR}\left(75 \mathrm{MHz}, \mathrm{CDCl}_{3}\right) \delta 139.9,128.5,128.2,126.2,109.7,72.1,69.0,45.4,43.7$, 33.7, 25.0.

IR (neat) 3510, 3027, 2948, 1603, 1496, 1454, 1110, 1014, 942, $701 \mathrm{~cm}^{-1}$.

HRMS (EI) m/e $\left(\mathrm{M}^{+}\right)$calcd for $\mathrm{C}_{13} \mathrm{H}_{16} \mathrm{O}_{2}$ : 204.1150, found: 204.1152 .

Determination of the relative stereochemistry via independent synthesis.
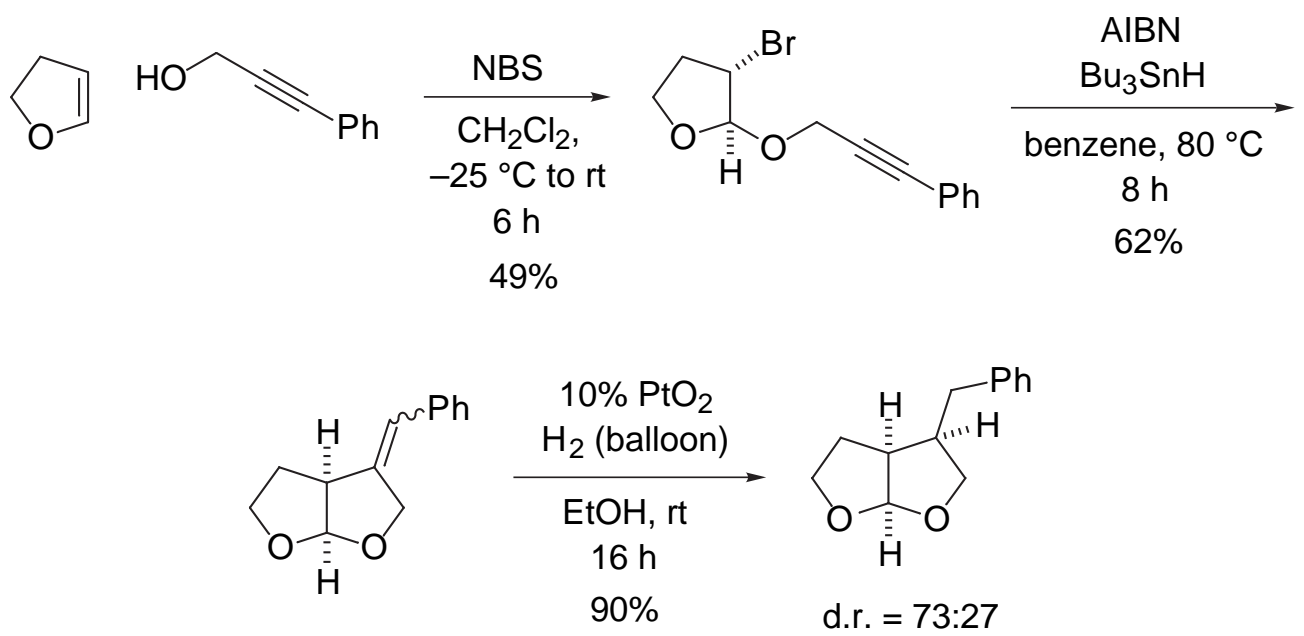

3-Bromo-2-(3-phenylprop-2-ynyloxy)tetrahydrofuran. Into an oven-dried roundbottom flask was added $\mathrm{N}$-bromosuccinimide $(3.60 \mathrm{~g}, 20.4 \mathrm{mmol})$ and dry $\mathrm{CH}_{2} \mathrm{Cl}_{2}(100$ $\mathrm{mL}$ ). The resulting suspension was cooled to -20 to $-25^{\circ} \mathrm{C}$, and 2,3-dihydrofuran (1.54 $\mathrm{mL}, 20.4 \mathrm{mmol}$ ) was added dropwise over a 20-min period. This mixture was stirred for $20 \mathrm{~min}$, and then 3-phenyl-2-propyn-1-ol ( $4.50 \mathrm{~mL}, 36.8 \mathrm{mmol})$ was added dropwise over a 30-min period. The reaction mixture was warmed to room temperature over a 6-h period, and then the reaction mixture was concentrated under reduced pressure. The resulting oil was taken up in $\mathrm{Et}_{2} \mathrm{O}(30 \mathrm{~mL})$ and poured into a separatory funnel that contained $\mathrm{H}_{2} \mathrm{O}(150 \mathrm{~mL})$. The aqueous layer was extracted with $\mathrm{Et}_{2} \mathrm{O}(3 \times 30 \mathrm{~mL})$, and the combined organic layers were washed with brine $(50 \mathrm{~mL})$, dried over $\mathrm{MgSO}_{4}$, filtered, and concentrated under reduced pressure. Purification by column chromatography (eluant: 90:10 hexanes: $\mathrm{Et}_{2} \mathrm{O}$ ) furnished $2.83 \mathrm{~g}(49 \%)$ of the desired product as a clear oil.

$\mathrm{R}_{f}=0.50$ (80:20 hexanes: $\left.\mathrm{Et}_{2} \mathrm{O}\right)$.

${ }^{1} \mathrm{H}$ NMR $\left(300 \mathrm{MHz}, \mathrm{CDCl}_{3}\right)$ d 7.49-7.46 (2H, m), 7.34-7.31 (3H, m), $5.49(1 \mathrm{H}, \mathrm{s}), 4.45$ $(2 \mathrm{H}, \mathrm{d}, J=4.0 \mathrm{~Hz}), 4.32(1 \mathrm{H}, \mathrm{d}, J=6.0 \mathrm{~Hz}), 4.21(1 \mathrm{H}, \mathrm{td}, J=8.5,7.5 \mathrm{~Hz}), 4.10(1 \mathrm{H}, \mathrm{td}, J=$ 8.5, 3.0 Hz), 2.74-2.61 (1H, m), 2.27-2.19 $(1 \mathrm{H}, \mathrm{m})$.

${ }^{13} \mathrm{C} \mathrm{NMR}\left(75 \mathrm{MHz}, \mathrm{CDCl}_{3}\right) \delta$ 131.7, 128.4, 128.2, 122.2, 106.8, 86.2, 84.2, 66.9, 54.6, 49.8, 33.6.

IR (neat) 2899, 2240, 1599, 1490, 1442, 1335, 1198, 1027, 757, $691 \mathrm{~cm}^{-1}$.

HRMS (EI) $m / e\left(\mathrm{M}^{+}\right)$calcd for $\mathrm{C}_{13} \mathrm{H}_{13} \mathrm{BrO}_{2}: 280.0099$, found: 280.0093 .

3-Benzylhexahydrofuro[2,3-b]furan. A solution of 3-bromo-2-(3-phenylprop-2ynyloxy)tetrahydrofuran (1.27 g, $4.50 \mathrm{mmol}), 2$,' '-azobisisobutyronitrile (AIBN; $37 \mathrm{mg}$, $0.23 \mathrm{mmol})$, and $\mathrm{Bu}_{3} \mathrm{SnH}(1.33 \mathrm{~mL}, 5.00 \mathrm{mmol})$ in benzene $(50 \mathrm{~mL})$ was heated to $80^{\circ} \mathrm{C}$ 
for $8 \mathrm{~h}$. The cooled reaction mixture was concentrated under reduced pressure and purified by column chromatography (eluant: $80: 20$ hexanes: $\mathrm{E}_{2} \mathrm{O}$ ), which furnished 563 $\mathrm{mg}(62 \%)$ of a clear oil that was used directly in the next step.

A suspension of the above compound $(200 \mathrm{mg}, 1.00 \mathrm{mmol})$ and $\mathrm{PtO}_{2}(25 \mathrm{mg}, 0.10$ $\mathrm{mmol})$ in $\mathrm{EtOH}(5 \mathrm{~mL})$ was stirred at room temperature under a hydrogen atmosphere (balloon) for $16 \mathrm{~h}$. The reaction mixture was filtered through a pad of celite on a sintered glass funnel ( $\mathrm{Et}_{2} \mathrm{O}$ washings), and then the filtrate was concentrated under reduced pressure. GC analysis revealed a 73:27 ratio of diastereomers. The reaction mixture was purified by column chromatography (eluant: 80:20 hexanes: $\mathrm{Et}_{2} \mathrm{O}$ ), which furnished $184 \mathrm{mg}(90 \%)$ of a clear oil. The major diastereomer is identical (GC and ${ }^{1} \mathrm{H}$ NMR) with the major diastereomer 4 from the cyclization/cross-coupling reaction illustrated in eq 4 . Based on related hydrogenations of methylenehexahydrofuro[2,3$b]$ furan ${ }^{8}$ and the expected delivery of hydrogen from the less hindered convex face, we have assigned the major diastereomer as the $3 R^{*}, 3 a S^{*}, 6 \mathrm{a} R^{*}$ (endo) compound.

Ligand effect on the nickel-catalyzed reaction of alkyl bromide 1 (eq 4; determined by GC analysis):

\begin{tabular}{|c|c|c|c|}
\hline Entry & Ligand & Yield $(\%)$ & d.r. \\
\hline 1 & 2,2'-bipyridine & 60 & 67:33 \\
\hline 2 & bathophenanthroline & 52 & 67:33 \\
\hline 3 & 4,4'-di-t-butyl-2,2'-bipyridine & 61 & $67: 33$ \\
\hline 4 & 4,4'-diphenyl-2,2'-bipyridine & 53 & 67:33 \\
\hline 5 & 4,4'-dimethoxy-2,2'-bipyridine & 57 & $67: 33$ \\
\hline
\end{tabular}

Ligand effect on the nickel-catalyzed reaction of alkyl bromide 2 (eq 4; determined by GC analysis):

\begin{tabular}{|c|c|c|}
\hline Entry & Ligand & $\underline{\text { Yield }(\%)}$ \\
\hline 1 & 2,2'-bipyridine & 53 \\
\hline 2 & bathophenanthroline & 21 \\
\hline 3 & 4,4'-di-t-butyl-2,2'-bipyridine & 40 \\
\hline 4 & 4,4'-diphenyl-2,2'-bipyridine & 21 \\
\hline 5 & 4,4'-dimethoxy-2,2'-bipyridine & 43 \\
\hline
\end{tabular}




\section{Crystal Structure of the Product of Table 3, Entry 1.}

Table 1. Crystal data and structure refinement for 04134dpm.

Identification code

Empirical formula

Formula weight

Temperature

Wavelength

Crystal system

Space group

Unit cell dimensions

Volume

Z

Density (calculated)

Absorption coefficient

$\mathrm{F}(000)$

Crystal size

Theta range for data collection

Index ranges

Reflections collected

Independent reflections

Completeness to theta $=28.29^{\circ}$

Refinement method

Data / restraints / parameters

Goodness-of-fit on $\mathrm{F}^{2}$

Final $\mathrm{R}$ indices [I $>2$ sigma(I)]

$\mathrm{R}$ indices (all data)

Absolute structure parameter

Largest diff. peak and hole 04134dpm

C34 H52 O

476.76

193(2) K

$0.71073 \AA$

monoclinic

P2(1)

$\mathrm{a}=16.275(3) \AA \quad \alpha=90^{\circ}$.

$\mathrm{b}=9.4620(19) \AA$

$\beta=95.45(3)^{\circ}$.

$\mathrm{c}=18.925(4) \AA$

2901.2(10) $\AA^{3}$

4

$1.092 \mathrm{Mg} / \mathrm{m}^{3}$

$0.063 \mathrm{~mm}^{-1}$

1056

$0.31 \times 0.38 \times 0.1 \mathrm{~mm}^{3}$

1.08 to $28.29^{\circ}$.

$-21<=\mathrm{h}<=19,-12<=\mathrm{k}<=12,-25<=1<=16$

21748

$13996[\mathrm{R}$ (int) $=0.0908]$

$99.8 \%$

Full-matrix least-squares on $\mathrm{F}^{2}$

13996 / 134 / 659

0.802

$\mathrm{R} 1=0.0625, \mathrm{wR} 2=0.1298$

$\mathrm{R} 1=0.1128, \mathrm{wR} 2=0.1508$

$-0.2(18)$

0.200 and -0.212 e. $\AA^{-3}$ 


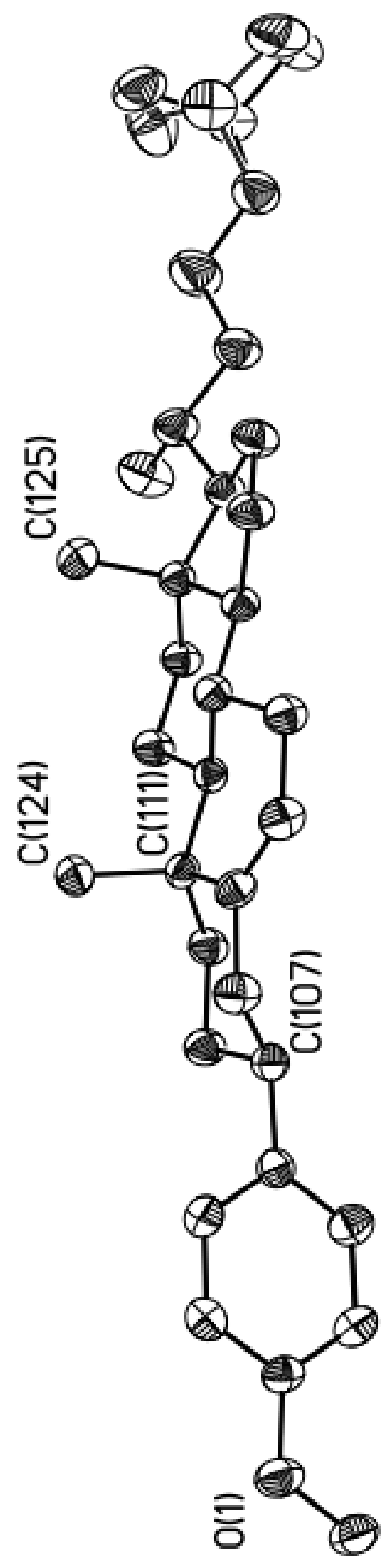

S-18 
S-19 
Table 2. Atomic coordinates ( $\times 10^{4}$ ) and equivalent isotropic displacement parameters $\left(\AA^{2} \times 10^{3}\right)$ for $04134 \mathrm{dpm}$. U(eq) is defined as one third of the trace of the orthogonalized Uij tensor.

\begin{tabular}{|c|c|c|c|c|}
\hline & $\mathrm{x}$ & $\mathrm{y}$ & $\mathrm{z}$ & $\mathrm{U}(\mathrm{eq})$ \\
\hline $\mathrm{O}(1)$ & $6156(1)$ & $5158(2)$ & 2812(1) & $57(1)$ \\
\hline$C(100)$ & 6592(2) & $3884(4)$ & $2955(2)$ & $63(1)$ \\
\hline$C(101)$ & $5357(2)$ & 5054(3) & $2510(1)$ & $43(1)$ \\
\hline$C(102)$ & $4974(2)$ & 3799(3) & $2307(2)$ & $50(1)$ \\
\hline$C(103)$ & $4930(2)$ & $6297(3)$ & $2405(2)$ & $57(1)$ \\
\hline$C(104)$ & $4118(2)$ & 6292(3) & $2112(2)$ & $58(1)$ \\
\hline$C(105)$ & $4156(2)$ & $3818(3)$ & $2011(2)$ & $52(1)$ \\
\hline$C(106)$ & $3709(2)$ & 5063(3) & 1908(1) & $44(1)$ \\
\hline$C(107)$ & $2806(2)$ & $5025(3)$ & 1640(1) & $45(1)$ \\
\hline$C(108)$ & $2244(2)$ & 5112(3) & $2251(1)$ & $48(1)$ \\
\hline$C(109)$ & $2541(2)$ & 6185(3) & $1101(2)$ & $52(1)$ \\
\hline$C(110)$ & $1639(2)$ & $6076(3)$ & $832(1)$ & $43(1)$ \\
\hline$C(111)$ & $1019(2)$ & 5994(3) & $1388(1)$ & $39(1)$ \\
\hline$C(112)$ & $1338(2)$ & 4930(3) & 1973(1) & $45(1)$ \\
\hline$C(113)$ & $1411(2)$ & 6048(3) & $136(2)$ & $50(1)$ \\
\hline$C(114)$ & $548(2)$ & 5952(3) & $-199(1)$ & $48(1)$ \\
\hline$C(115)$ & $-97(2)$ & $6215(3)$ & $326(1)$ & $39(1)$ \\
\hline$C(116)$ & $170(2)$ & $5475(3)$ & $1039(1)$ & $37(1)$ \\
\hline$C(117)$ & $-528(2)$ & $5546(3)$ & 1540(1) & $46(1)$ \\
\hline$C(118)$ & $-1364(2)$ & $5020(3)$ & $1202(1)$ & $46(1)$ \\
\hline$C(119)$ & $-1636(2)$ & $5832(3)$ & $513(1)$ & $41(1)$ \\
\hline$C(120)$ & $-934(2)$ & $5660(3)$ & $28(1)$ & $40(1)$ \\
\hline$C(121)$ & $-1303(2)$ & $6205(3)$ & $-690(1)$ & $52(1)$ \\
\hline$C(122)$ & $-2207(2)$ & $5695(4)$ & $-735(2)$ & $56(1)$ \\
\hline$C(123)$ & $-2362(2)$ & 5175(3) & $26(1)$ & $47(1)$ \\
\hline$C(124)$ & 953(2) & 7474(3) & $1724(2)$ & $52(1)$ \\
\hline$C(125)$ & $-1810(2)$ & 7389(3) & $668(2)$ & $56(1)$ \\
\hline$C(126)$ & $-3264(2)$ & $5443(4)$ & $179(2)$ & $57(1)$ \\
\hline$C(127)$ & $-3439(2)$ & 5011(5) & $931(2)$ & $81(1)$ \\
\hline$C(128)$ & $-3855(2)$ & $4666(4)$ & $-378(2)$ & $67(1)$ \\
\hline
\end{tabular}




\begin{tabular}{|c|c|c|c|c|}
\hline$C(129)$ & $-4745(2)$ & $5077(6)$ & $-427(2)$ & $98(1)$ \\
\hline$C(130)$ & $-5251(2)$ & $4474(4)$ & $-1081(2)$ & $75(1)$ \\
\hline$C(131)$ & $-6173(4)$ & $4544(8)$ & $-1136(4)$ & $65(2)$ \\
\hline$C(132)$ & $-6563(10)$ & $3980(20)$ & $-1838(7)$ & $85(4)$ \\
\hline$C(133)$ & $-6427(6)$ & $6086(9)$ & $-1028(6)$ & $93(3)$ \\
\hline$C(31 B)$ & $-6042(6)$ & $5261(16)$ & $-1315(6)$ & $84(3)$ \\
\hline$C(32 B)$ & $-6500(12)$ & $4460(30)$ & $-1921(10)$ & $98(8)$ \\
\hline $\mathrm{C}(33 \mathrm{~B})$ & $-6570(8)$ & $5350(20)$ & $-679(8)$ & $145(7)$ \\
\hline $\mathrm{O}(2)$ & $-1565(1)$ & 5801(3) & $3658(1)$ & $64(1)$ \\
\hline$C(200)$ & $-1842(2)$ & $6792(4)$ & $3118(2)$ & $61(1)$ \\
\hline$C(201)$ & $-739(2)$ & $5855(4)$ & $3906(2)$ & $49(1)$ \\
\hline$C(202)$ & $-185(2)$ & $6836(3)$ & $3699(2)$ & $54(1)$ \\
\hline$C(203)$ & $-472(2)$ & $4843(4)$ & $4395(2)$ & $63(1)$ \\
\hline$C(204)$ & $634(2)$ & 6793(3) & $3989(2)$ & $52(1)$ \\
\hline$C(205)$ & $348(2)$ & $4800(4)$ & $4675(2)$ & $58(1)$ \\
\hline$C(206)$ & $920(2)$ & 5784(3) & $4476(1)$ & $43(1)$ \\
\hline$C(207)$ & $1824(2)$ & $5770(3)$ & $4766(1)$ & $40(1)$ \\
\hline$C(208)$ & $2322(2)$ & $4593(3)$ & $4444(1)$ & $41(1)$ \\
\hline$C(209)$ & 1969(1) & $5641(3)$ & $5577(1)$ & $43(1)$ \\
\hline$C(210)$ & $3231(2)$ & $4688(3)$ & $4680(1)$ & $37(1)$ \\
\hline$C(211)$ & $3469(2)$ & $4758(3)$ & $5485(1)$ & $35(1)$ \\
\hline$C(212)$ & $2885(1)$ & $5802(3)$ & $5826(1)$ & $39(1)$ \\
\hline$C(213)$ & $3786(2)$ & $4720(3)$ & $4208(1)$ & $40(1)$ \\
\hline $\mathrm{C}(214)$ & $4697(2)$ & $4818(3)$ & $4373(1)$ & $41(1)$ \\
\hline$C(215)$ & $4977(2)$ & $4585(3)$ & $5160(1)$ & $35(1)$ \\
\hline$C(216)$ & $4376(1)$ & $5301(3)$ & $5629(1)$ & $35(1)$ \\
\hline$C(217)$ & $4712(1)$ & $5239(3)$ & $6418(1)$ & $40(1)$ \\
\hline$C(218)$ & $5604(1)$ & $5764(3)$ & $6573(1)$ & $39(1)$ \\
\hline$C(219)$ & $6196(1)$ & $4986(3)$ & $6126(1)$ & $36(1)$ \\
\hline$C(220)$ & $5844(1)$ & $5159(3)$ & $5343(1)$ & $36(1)$ \\
\hline$C(221)$ & $6538(2)$ & $4647(3)$ & $4918(1)$ & $43(1)$ \\
\hline$C(222)$ & 7334(1) & 5091(3) & $5368(1)$ & $41(1)$ \\
\hline$C(223)$ & $7071(1)$ & $5643(3)$ & $6088(1)$ & $36(1)$ \\
\hline$C(224)$ & $3370(2)$ & $3279(3)$ & $5802(2)$ & $45(1)$ \\
\hline$C(225)$ & $6285(2)$ & $3422(3)$ & $6343(2)$ & $49(1)$ \\
\hline$C(226)$ & $7740(2)$ & $5402(3)$ & $6704(1)$ & $41(1)$ \\
\hline
\end{tabular}




$\begin{array}{lrlll}\mathrm{C}(227) & 7457(2) & 5858(4) & 7418(2) & 63(1) \\ \mathrm{C}(228) & 8544(2) & 6171(3) & 6573(2) & 45(1) \\ \mathrm{C}(229) & 9266(2) & 5896(3) & 7124(2) & 51(1) \\ \mathrm{C}(230) & 10069(2) & 6595(3) & 6967(2) & 50(1) \\ \mathrm{C}(231) & 10732(2) & 6622(3) & 7591(2) & 50(1) \\ \mathrm{C}(232) & 10954(2) & 5178(4) & 7882(2) & 70(1) \\ \mathrm{C}(233) & 11494(2) & 7419(4) & 7403(2) & 61(1)\end{array}$


Table 3. Bond lengths $[\AA]$ and angles $\left[{ }^{\circ}\right]$ for $04134 \mathrm{dpm}$.

\begin{tabular}{ll}
\hline $\mathrm{O}(1)-\mathrm{C}(101)$ & $1.374(3)$ \\
$\mathrm{O}(1)-\mathrm{C}(100)$ & $1.412(4)$ \\
$\mathrm{C}(101)-\mathrm{C}(103)$ & $1.371(4)$ \\
$\mathrm{C}(101)-\mathrm{C}(102)$ & $1.378(4)$ \\
$\mathrm{C}(102)-\mathrm{C}(105)$ & $1.395(4)$ \\
$\mathrm{C}(103)-\mathrm{C}(104)$ & $1.383(4)$ \\
$\mathrm{C}(104)-\mathrm{C}(106)$ & $1.377(4)$ \\
$\mathrm{C}(105)-\mathrm{C}(106)$ & $1.388(4)$ \\
$\mathrm{C}(106)-\mathrm{C}(107)$ & $1.509(4)$ \\
$\mathrm{C}(107)-\mathrm{C}(109)$ & $1.531(4)$ \\
$\mathrm{C}(107)-\mathrm{C}(108)$ & $1.542(4)$ \\
$\mathrm{C}(108)-\mathrm{C}(112)$ & $1.528(3)$ \\
$\mathrm{C}(109)-\mathrm{C}(110)$ & $1.511(4)$ \\
$\mathrm{C}(110)-\mathrm{C}(113)$ & $1.332(4)$ \\
$\mathrm{C}(110)-\mathrm{C}(111)$ & $1.527(4)$ \\
$\mathrm{C}(111)-\mathrm{C}(124)$ & $1.547(4)$ \\
$\mathrm{C}(111)-\mathrm{C}(116)$ & $1.554(3)$ \\
$\mathrm{C}(111)-\mathrm{C}(112)$ & $1.549(4)$ \\
$\mathrm{C}(113)-\mathrm{C}(114)$ & $1.489(4)$ \\
$\mathrm{C}(114)-\mathrm{C}(115)$ & $1.534(4)$ \\
$\mathrm{C}(115)-\mathrm{C}(120)$ & $1.517(3)$ \\
$\mathrm{C}(115)-\mathrm{C}(116)$ & $1.545(3)$ \\
$\mathrm{C}(116)-\mathrm{C}(117)$ & $1.549(3)$ \\
$\mathrm{C}(117)-\mathrm{C}(118)$ & $1.530(4)$ \\
$\mathrm{C}(118)-\mathrm{C}(119)$ & $1.542(4)$ \\
$\mathrm{C}(119)-\mathrm{C}(125)$ & $1.534(4)$ \\
$\mathrm{C}(119)-\mathrm{C}(120)$ & $1.541(3)$ \\
$\mathrm{C}(119)-\mathrm{C}(123)$ & $1.557(4)$ \\
$\mathrm{C}(120)-\mathrm{C}(121)$ & $1.522(3)$ \\
$\mathrm{C}(121)-\mathrm{C}(122)$ & $1.543(4)$ \\
$\mathrm{C}(122)-\mathrm{C}(123)$ & $1.566(4)$ \\
$\mathrm{C}(123)-\mathrm{C}(126)$ & $1.543(4)$ \\
$\mathrm{C}(126)-\mathrm{C}(127)$ & $1.533(4)$ \\
$\mathrm{C}(126)-\mathrm{C}(128)$ & $1.545(4)$ \\
\end{tabular}




$\begin{array}{ll}\mathrm{C}(128)-\mathrm{C}(129) & 1.495(4) \\ \mathrm{C}(129)-\mathrm{C}(130) & 1.530(5) \\ \mathrm{C}(130)-\mathrm{C}(131) & 1.497(7) \\ \mathrm{C}(130)-\mathrm{C}(31 \mathrm{~B}) & 1.517(10) \\ \mathrm{C}(131)-\mathrm{C}(132) & 1.516(9) \\ \mathrm{C}(131)-\mathrm{C}(133) & 1.535(9) \\ \mathrm{C}(31 \mathrm{~B})-\mathrm{C}(32 \mathrm{~B}) & 1.510(12) \\ \mathrm{C}(31 \mathrm{~B})-\mathrm{C}(33 \mathrm{~B}) & 1.546(13) \\ \mathrm{O}(2)-\mathrm{C}(201) & 1.381(3) \\ \mathrm{O}(2)-\mathrm{C}(200) & 1.428(4) \\ \mathrm{C}(201)-\mathrm{C}(203) & 1.372(4) \\ \mathrm{C}(201)-\mathrm{C}(202) & 1.377(4) \\ \mathrm{C}(202)-\mathrm{C}(204) & 1.394(4) \\ \mathrm{C}(203)-\mathrm{C}(205) & 1.389(4) \\ \mathrm{C}(204)-\mathrm{C}(206) & 1.377(4) \\ \mathrm{C}(205)-\mathrm{C}(206) & 1.393(4) \\ \mathrm{C}(206)-\mathrm{C}(207) & 1.521(3) \\ \mathrm{C}(207)-\mathrm{C}(209) & 1.535(4) \\ \mathrm{C}(207)-\mathrm{C}(208) & 1.536(4) \\ \mathrm{C}(208)-\mathrm{C}(210) & 1.508(3) \\ \mathrm{C}(209)-\mathrm{C}(212) & 1.528(3) \\ \mathrm{C}(210)-\mathrm{C}(213) & 1.329(3) \\ \mathrm{C}(210)-\mathrm{C}(211) & 1.537(3) \\ \mathrm{C}(211)-\mathrm{C}(224) & 1.538(4) \\ \mathrm{C}(211)-\mathrm{C}(212) & 1.554(3) \\ \mathrm{C}(211)-\mathrm{C}(216) & 1.562(3) \\ \mathrm{C}(213)-\mathrm{C}(214) & 1.489(3) \\ \mathrm{C}(214)-\mathrm{C}(215) & 1.532(3) \\ \mathrm{C}(215)-\mathrm{C}(220) & 1.521(3) \\ \mathrm{C}(215)-\mathrm{C}(216) & 1.539(3) \\ \mathrm{C}(216)-\mathrm{C}(217) & 1.541(3) \\ \mathrm{C}(217)-\mathrm{C}(218) & 1.535(3) \\ \mathrm{C}(218)-\mathrm{C}(219) & 1.529(4) \\ \mathrm{C}(219)-\mathrm{C}(225) & 1.539(4) \\ \mathrm{C}(219)-\mathrm{C}(220) & 1.546(3) \\ \mathrm{C}(219)-\mathrm{C}(223) & 1.561(3)\end{array}$




$\begin{array}{lr}C(220)-C(221) & 1.526(3) \\ C(221)-C(222) & 1.540(3) \\ C(222)-C(223) & 1.557(4) \\ C(223)-C(226) & 1.535(3) \\ C(226)-C(227) & 1.530(4) \\ C(226)-C(228) & 1.538(3) \\ C(228)-C(229) & 1.518(3) \\ C(229)-C(230) & 1.518(3) \\ C(230)-C(231) & 1.523(4) \\ C(231)-C(232) & 1.504(4) \\ C(231)-C(233) & 1.523(4) \\ C(101)-O(1)-C(100) & 117.2(2) \\ O(1)-C(101)-C(103) & 116.5(3) \\ O(1)-C(101)-C(102) & 124.2(3) \\ C(103)-C(101)-C(102) 119.3(2) \\ C(101)-C(102)-C(105) 119.3(3) \\ C(101)-C(103)-C(104) 120.4(3) \\ C(106)-C(104)-C(103) 122.2(3) \\ C(106)-C(105)-C(102) 122.3(3) \\ C(104)-C(106)-C(105) 116.4(2) \\ C(104)-C(106)-C(107) 122.9(3) \\ C(105)-C(106)-C(107) 120.5(3) \\ C(106)-C(107)-C(109) 114.3(2) \\ C(106)-C(107)-C(108) 112.0(2) \\ C(109)-C(107)-C(108) 108.2(2) \\ C(112)-C(108)-C(107) 110.9(2) \\ C(110)-C(109)-C(107) 112.3(2) \\ C(113)-C(110)-C(109) 120.3(2) \\ C(113)-C(110)-C(111) 122.7(2) \\ C(109)-C(110)-C(111) 117.0(2) \\ C(110)-C(111)-C(124) 108.3(2) \\ C(110)-C(111)-C(116) 110.0(2) \\ C(124)-C(111)-C(116) 111.5(2) \\ C(110)-C(111)-C(112) 109.2(2) \\ C(124)-C(111)-C(112) 109.0(2)\end{array}$


$\mathrm{C}(116)-\mathrm{C}(111)-\mathrm{C}(112) 108.8(2)$
$\mathrm{C}(108)-\mathrm{C}(112)-\mathrm{C}(111) 114.8(2)$
$\mathrm{C}(110)-\mathrm{C}(113)-\mathrm{C}(114) 125.8(3)$
$\mathrm{C}(113)-\mathrm{C}(114)-\mathrm{C}(115) 112.9(2)$
$\mathrm{C}(120)-\mathrm{C}(115)-\mathrm{C}(114) 110.4(2)$
$\mathrm{C}(120)-\mathrm{C}(115)-\mathrm{C}(116) 109.6(2)$
$\mathrm{C}(114)-\mathrm{C}(115)-\mathrm{C}(116) 109.8(2)$
$\mathrm{C}(117)-\mathrm{C}(116)-\mathrm{C}(115) 110.7(2)$
$\mathrm{C}(117)-\mathrm{C}(116)-\mathrm{C}(111) 113.6(2)$
$\mathrm{C}(115)-\mathrm{C}(116)-\mathrm{C}(111) 113.2(2)$
$\mathrm{C}(118)-\mathrm{C}(117)-\mathrm{C}(116) 113.9(2)$
$\mathrm{C}(117)-\mathrm{C}(118)-\mathrm{C}(119) 111.4(2)$
$\mathrm{C}(125)-\mathrm{C}(119)-\mathrm{C}(120) 112.1(2)$
$\mathrm{C}(125)-\mathrm{C}(119)-\mathrm{C}(118) 111.1(2)$
$\mathrm{C}(120)-\mathrm{C}(119)-\mathrm{C}(118) 106.3(2)$
$\mathrm{C}(125)-\mathrm{C}(119)-\mathrm{C}(123) 110.7(2)$
$\mathrm{C}(120)-\mathrm{C}(119)-\mathrm{C}(123) 99.8(2)$
$\mathrm{C}(118)-\mathrm{C}(119)-\mathrm{C}(123) 116.2(2)$
$\mathrm{C}(121)-\mathrm{C}(120)-\mathrm{C}(115) 118.6(2)$
$\mathrm{C}(121)-\mathrm{C}(120)-\mathrm{C}(119) 104.1(2)$
$\mathrm{C}(115)-\mathrm{C}(120)-\mathrm{C}(119) 115.6(2)$
$\mathrm{C}(120)-\mathrm{C}(121)-\mathrm{C}(122) 103.7(2)$
$\mathrm{C}(121)-\mathrm{C}(122)-\mathrm{C}(123) 106.4(2)$
$\mathrm{C}(126)-\mathrm{C}(123)-\mathrm{C}(122) 111.1(2)$
$\mathrm{C}(126)-\mathrm{C}(123)-\mathrm{C}(119) 120.4(2)$
$\mathrm{C}(122)-\mathrm{C}(123)-\mathrm{C}(119) 103.8(2)$
$\mathrm{C}(127)-\mathrm{C}(126)-\mathrm{C}(123) 113.2(2)$
$\mathrm{C}(127)-\mathrm{C}(126)-\mathrm{C}(128) 110.4(3)$
$\mathrm{C}(123)-\mathrm{C}(126)-\mathrm{C}(128) 109.6(3)$
$\mathrm{C}(129)-\mathrm{C}(128)-\mathrm{C}(126) 117.1(3)$
$\mathrm{C}(128)-\mathrm{C}(129)-\mathrm{C}(130) 113.3(3)$
$\mathrm{C}(131)-\mathrm{C}(130)-\mathrm{C}(31 \mathrm{~B}) 30.6(4)$
$\mathrm{C}(131)-\mathrm{C}(130)-\mathrm{C}(129) 119.9(4)$
$\mathrm{C}(31 \mathrm{~B})-\mathrm{C}(130)-\mathrm{C}(129) 115.5(6)$
$\mathrm{C}(130)-\mathrm{C}(131)-\mathrm{C}(132) 112.2(9)$
$\mathrm{C}(130)-\mathrm{C}(131)-\mathrm{C}(133) 108.3(7)$ 


$C(132)-C(131)-C(133) 111.0(9)$
$C(32 B)-C(31 B)-C(130) 108.7(11)$
$C(32 B)-C(31 B)-C(33 B) 110.3(12)$
$C(130)-C(31 B)-C(33 B) 108.5(11)$
$C(201)-O(2)-C(200) 116.8(2)$
$C(203)-C(201)-C(202) 119.2(3)$
$C(203)-C(201)-O(2) 116.0(3)$
$C(202)-C(201)-O(2) 124.7(3)$
$C(201)-C(202)-C(204) 119.7(3)$
$C(201)-C(203)-C(205) 120.6(3)$
$C(206)-C(204)-C(202) 122.3(3)$
$C(206)-C(205)-C(203) 121.3(3)$
$C(204)-C(206)-C(205) 116.8(3)$
$C(204)-C(206)-C(207) 120.1(2)$
$C(205)-C(206)-C(207) 123.1(3)$
$C(206)-C(207)-C(209) 114.3(2)$
$C(206)-C(207)-C(208) 113.0(2)$
$C(209)-C(207)-C(208) 107.6(2)$
$C(210)-C(208)-C(207) 112.3(2)$
$C(212)-C(209)-C(207) 110.7(2)$
$C(213)-C(210)-C(208) 120.9(2)$
$C(213)-C(210)-C(211) 122.8(2)$
$C(208)-C(210)-C(211) 116.3(2)$
$C(210)-C(211)-C(224) 108.8(2)$
$C(210)-C(211)-C(212) 109.4(2)$
$C(224)-C(211)-C(212) 108.9(2)$
$C(210)-C(211)-C(216) 109.45(19)$
$C(224)-C(211)-C(216) 111.3(2)$
$C(212)-C(211)-C(216) 108.9(2)$
$C(209)-C(212)-C(211) 115.3(2)$
$C(210)-C(213)-C(214) 126.0(2)$
$C(213)-C(214)-C(215) 113.1(2)$
$C(220)-C(215)-C(214) 110.7(2)$
$C(220)-C(215)-C(216) 109.7(2)$
$C(214)-C(215)-C(216) 110.6(2)$
$C(215)-C(216)-C(217) 110.9(2)$
C)




$$
\begin{aligned}
& C(215)-C(216)-C(211) 113.3(2) \\
& C(217)-C(216)-C(211) 113.57(19) \\
& C(218)-C(217)-C(216) 114.5(2) \\
& C(217)-C(218)-C(219) 111.9(2) \\
& C(218)-C(219)-C(225) 111.3(2) \\
& C(218)-C(219)-C(220) 106.44(19) \\
& C(225)-C(219)-C(220) 111.9(2) \\
& C(218)-C(219)-C(223) 117.4(2) \\
& C(225)-C(219)-C(223) 109.4(2) \\
& C(220)-C(219)-C(223) 99.82(19) \\
& C(215)-C(220)-C(221) 118.9(2) \\
& C(215)-C(220)-C(219) 115.6(2) \\
& C(221)-C(220)-C(219) 104.2(2) \\
& C(220)-C(221)-C(222) 104.4(2) \\
& C(221)-C(222)-C(223) 106.74(19) \\
& C(226)-C(223)-C(222) 112.4(2) \\
& C(226)-C(223)-C(219) 119.4(2) \\
& C(22)-C(223)-C(219) 103.6(2) \\
& C(227)-C(226)-C(223) 112.2(2) \\
& C(227)-C(226)-C(228) 110.0(2) \\
& C(223)-C(226)-C(228) 111.1(2) \\
& C(229)-C(228)-C(226) 115.0(2) \\
& C(230)-C(229)-C(228) 114.5(2) \\
& C(229)-C(230)-C(231) 114.5(2) \\
& C(232)-C(231)-C(230) 113.3(2) \\
& C(232)-C(231)-C(233) 111.3(3) \\
& C(230)-C(231)-C(233) 111.1(2)
\end{aligned}
$$

Symmetry transformations used to generate equivalent atoms: 
Table 4. Anisotropic displacement parameters $\left(\AA^{2} \times 10^{3}\right)$ for $04134 \mathrm{dpm}$. The anisotropic displacement factor exponent takes the form: $-2 p^{2}\left[h^{2} a^{* 2} U^{11}+\ldots+2 h k\right.$ $a^{*} b^{*} U^{12}$ ]

\begin{tabular}{|c|c|c|c|c|c|}
\hline U11 & $\mathrm{U}^{22}$ & U33 & $U^{23}$ & $\mathrm{U}^{13}$ & $\mathrm{U}^{12}$ \\
\hline $\mathrm{O}(1) 40(1)$ & $73(1)$ & $57(1)$ & $1(1)$ & $4(1)$ & $4(1)$ \\
\hline $\mathrm{C}(100) 47(2)$ & $77(2)$ & $64(2)$ & $-1(2)$ & $-3(2)$ & $15(2)$ \\
\hline$C(101) 36(2)$ & $60(2)$ & $35(1)$ & $2(1)$ & $8(1)$ & $4(1)$ \\
\hline$C(102) 42(2)$ & $50(2)$ & $58(2)$ & $7(2)$ & $9(2)$ & $6(1)$ \\
\hline$C(103) 53(2)$ & $57(2)$ & $60(2)$ & $-15(2)$ & $-3(2)$ & $-1(2)$ \\
\hline$C(104) 52(2)$ & $60(2)$ & $59(2)$ & $-14(2)$ & $-1(2)$ & $15(2)$ \\
\hline $\mathrm{C}(105) 47(2)$ & $53(2)$ & $57(2)$ & $6(2)$ & $7(2)$ & $-1(1)$ \\
\hline$C(106) 46(2)$ & $51(2)$ & $35(1)$ & $-2(1)$ & $5(1)$ & $3(1)$ \\
\hline $\mathrm{C}(107) 42(2)$ & $53(2)$ & $40(2)$ & $-6(1)$ & $4(1)$ & $5(1)$ \\
\hline $\mathrm{C}(108) 44(2)$ & $63(2)$ & $36(1)$ & $1(2)$ & $1(1)$ & $6(1)$ \\
\hline $\mathrm{C}(109) 44(2)$ & $67(2)$ & $45(2)$ & $4(2)$ & $8(1)$ & $0(1)$ \\
\hline$C(110) 39(2)$ & $47(2)$ & $45(2)$ & $6(1)$ & $8(1)$ & $4(1)$ \\
\hline $\mathrm{C}(111) 38(1)$ & $42(2)$ & $38(1)$ & $-2(1)$ & $6(1)$ & $5(1)$ \\
\hline $\mathrm{C}(112) 44(2)$ & $57(2)$ & $34(1)$ & $0(1)$ & $4(1)$ & $1(1)$ \\
\hline$C(113) 45(2)$ & $65(2)$ & $42(2)$ & $8(2)$ & $16(1)$ & $4(1)$ \\
\hline$C(114) 46(2)$ & $62(2)$ & $36(1)$ & $4(1)$ & $7(1)$ & $5(1)$ \\
\hline$C(115) 41(2)$ & $42(2)$ & $35(1)$ & $3(1)$ & $6(1)$ & $3(1)$ \\
\hline$C(116) 41(2)$ & $38(2)$ & $34(1)$ & $-2(1)$ & $5(1)$ & $4(1)$ \\
\hline $\mathrm{C}(117) 44(2)$ & $64(2)$ & $30(1)$ & $3(1)$ & $6(1)$ & $3(1)$ \\
\hline $\mathrm{C}(118) 44(2)$ & $60(2)$ & $36(1)$ & $4(1)$ & $6(1)$ & $1(1)$ \\
\hline $\mathrm{C}(119) 40(1)$ & $47(2)$ & $36(1)$ & $1(1)$ & $4(1)$ & $3(1)$ \\
\hline $\mathrm{C}(120) 44(2)$ & $42(2)$ & $33(1)$ & $7(1)$ & $2(1)$ & $3(1)$ \\
\hline $\mathrm{C}(121) 53(2)$ & $66(2)$ & $35(2)$ & $10(1)$ & $2(1)$ & $5(2)$ \\
\hline$C(122) 53(2)$ & $74(2)$ & $41(2)$ & $5(2)$ & $-1(1)$ & $0(2)$ \\
\hline$C(123) 46(2)$ & $55(2)$ & $39(2)$ & $4(1)$ & $1(1)$ & $3(1)$ \\
\hline $\mathrm{C}(124) 47(2)$ & $49(2)$ & $59(2)$ & $-11(2)$ & $2(2)$ & $0(1)$ \\
\hline $\mathrm{C}(125) 48(2)$ & $58(2)$ & $61(2)$ & $-2(2)$ & $6(2)$ & $6(1)$ \\
\hline $\mathrm{C}(126) 40(2)$ & $75(2)$ & $57(2)$ & $1(2)$ & $5(1)$ & $2(1)$ \\
\hline $\mathrm{C}(127) 53(2)$ & 134(3) & $59(2)$ & $-2(2)$ & $16(2)$ & $-14(2)$ \\
\hline$C(128) 54(2)$ & $86(3)$ & $61(2)$ & $-2(2)$ & $2(2)$ & $0(2)$ \\
\hline
\end{tabular}




$\begin{array}{lccccc}\mathrm{C}(129) 56(2) & 148(4) & 90(3) & -34(3) & 0(2) & 6(2) \\ \mathrm{C}(130) 55(2) & 93(3) & 75(2) & -11(2) & 0(2) & -2(2) \\ \mathrm{C}(131) 53(3) & 80(5) & 64(4) & 18(4) & 11(3) & -10(3) \\ \mathrm{C}(132) 58(5) & 136(10) & 62(5) & -6(6) & 14(4) & -23(6) \\ \mathrm{C}(133) 62(5) & 113(6) & 103(7) & 3(5) & 1(5) & 26(5) \\ \mathrm{C}(31 \mathrm{~B}) 73(6) & 95(10) & 87(7) & 11(6) & 17(4) & -11(6) \\ \mathrm{C}(32 \mathrm{~B}) 35(6) & 170(20) & 89(9) & 1(10) & 4(6) & 7(9) \\ \mathrm{C}(33 \mathrm{~B}) 94(8) & 187(18) & 164(13) & -87(13) & 60(9) & -28(10) \\ \mathrm{O}(2) 33(1) & 90(2) & 69(1) & 4(1) & 0(1) & -2(1) \\ \mathrm{C}(200) 39(2) & 90(2) & 54(2) & -9(2) & 1(2) & 10(2) \\ \mathrm{C}(201) 32(2) & 68(2) & 47(2) & -6(2) & 3(1) & -3(1) \\ \mathrm{C}(202) 40(2) & 72(2) & 49(2) & 9(2) & 2(1) & 2(2) \\ \mathrm{C}(203) 42(2) & 77(2) & 68(2) & 13(2) & 7(2) & -15(2) \\ \mathrm{C}(204) 39(2) & 63(2) & 52(2) & 9(2) & 5(1) & -9(1) \\ \mathrm{C}(205) 44(2) & 69(2) & 62(2) & 14(2) & 0(2) & -6(2) \\ \mathrm{C}(206) 35(1) & 54(2) & 40(2) & -6(1) & 6(1) & -2(1) \\ \mathrm{C}(207) 36(1) & 45(2) & 40(1) & -2(1) & 5(1) & -8(1) \\ \mathrm{C}(208) 39(2) & 51(2) & 35(1) & -7(1) & 4(1) & -7(1) \\ \mathrm{C}(209) 34(1) & 53(2) & 43(2) & -7(1) & 9(1) & 1(1) \\ \mathrm{C}(210) 39(1) & 38(2) & 35(1) & -6(1) & 5(1) & -5(1) \\ \mathrm{C}(211) 34(1) & 40(2) & 32(1) & -4(1) & 6(1) & -2(1) \\ \mathrm{C}(212) 37(1) & 48(2) & 33(1) & -3(1) & 4(1) & -1(1) \\ \mathrm{C}(213) 40(1) & 48(2) & 31(1) & -4(1) & 3(1) & -4(1) \\ \mathrm{C}(214) 41(2) & 52(2) & 32(1) & -7(1) & 8(1) & -2(1) \\ \mathrm{C}(215) 36(1) & 37(1) & 33(1) & -1(1) & 6(1) & 0(1) \\ \mathrm{C}(216) 38(1) & 37(2) & 30(1) & 0(1) & 8(1) & 1(1) \\ \mathrm{C}(217) 34(1) & 55(2) & 31(1) & 0(1) & 5(1) & 2(1) \\ \mathrm{C}(218) 36(1) & 52(2) & 30(1) & -1(1) & 3(1) & 1(1) \\ \mathrm{C}(219) 32(1) & 41(1) & 35(1) & 5(1) & 3(1) & 3(1) \\ \mathrm{C}(220) 37(1) & 38(1) & 32(1) & -2(1) & 6(1) & 2(1) \\ \mathrm{C}(221) 39(2) & 49(2) & 43(2) & -6(1) & 11(1) & 2(1) \\ \mathrm{C}(222) 38(1) & 44(2) & 42(2) & 1(1) & 10(1) & 1(1) \\ \mathrm{C}(223) 33(1) & 36(1) & 39(1) & 5(1) & 3(1) & 4(1) \\ \mathrm{C}(224) 41(2) & 47(2) & 47(2) & 1(1) & 9(1) & -4(1) \\ \mathrm{C}(225) 43(2) & 49(2) & 55(2) & 9(1) & 7(1) & -3(1) \\ \mathrm{C}(226) 34(1) & 50(2) & 39(1) & 8(1) & 3(1) & 3(1) \\ & & & & & \end{array}$




$\begin{array}{lccccc}\mathrm{C}(227) 40(2) & 109(3) & 40(2) & 4(2) & -2(1) & -8(2) \\ \mathrm{C}(228) 35(1) & 56(2) & 45(2) & 5(1) & 1(1) & -1(1) \\ \mathrm{C}(229) 40(2) & 62(2) & 51(2) & 12(2) & -1(1) & -8(1) \\ \mathrm{C}(230) 40(2) & 67(2) & 44(2) & 4(2) & 5(1) & -8(1) \\ \mathrm{C}(231) 37(2) & 63(2) & 48(2) & -2(2) & 4(1) & -6(1) \\ \mathrm{C}(232) 45(2) & 79(2) & 84(3) & 13(2) & -10(2) & -3(2) \\ \mathrm{C}(233) 45(2) & 88(2) & 51(2) & -6(2) & 9(2) & -17(2)\end{array}$


Table 5. Hydrogen coordinates ( $\left.x 10^{4}\right)$ and isotropic displacement parameters $\left(\AA^{2} \mathrm{x}\right.$ $10^{3}$ ) for 04134dpm.

\begin{tabular}{|c|c|c|c|c|}
\hline & $x$ & $\mathrm{y}$ & $\mathrm{z}$ & $\mathrm{U}(\mathrm{eq})$ \\
\hline $\mathrm{H}(10 \mathrm{~A})$ & 7150 & 4097 & 3169 & 94 \\
\hline $\mathrm{H}(10 \mathrm{~B})$ & 6625 & 3362 & 2511 & 94 \\
\hline $\mathrm{H}(10 \mathrm{C})$ & 6303 & 3310 & 3284 & 94 \\
\hline $\mathrm{H}(102)$ & 5264 & 2930 & 2368 & 60 \\
\hline $\mathrm{H}(103)$ & 5193 & 7168 & 2534 & 69 \\
\hline $\mathrm{H}(104)$ & 3834 & 7166 & 2048 & 69 \\
\hline $\mathrm{H}(105)$ & 3896 & 2949 & 1874 & 63 \\
\hline $\mathrm{H}(107)$ & 2697 & 4095 & 1398 & 54 \\
\hline $\mathrm{H}(10 \mathrm{D})$ & 2403 & 4364 & 2604 & 57 \\
\hline $\mathrm{H}(10 \mathrm{E})$ & 2321 & 6038 & 2491 & 57 \\
\hline $\mathrm{H}(10 \mathrm{~F})$ & 2645 & 7120 & 1326 & 62 \\
\hline $\mathrm{H}(10 \mathrm{G})$ & 2879 & 6116 & 695 & 62 \\
\hline $\mathrm{H}(11 \mathrm{~A})$ & 997 & 5025 & 2377 & 54 \\
\hline $\mathrm{H}(11 \mathrm{~B})$ & 1258 & 3960 & 1783 & 54 \\
\hline $\mathrm{H}(113)$ & 1838 & 6093 & -172 & 60 \\
\hline $\mathrm{H}(11 \mathrm{C})$ & 458 & 5002 & -411 & 57 \\
\hline $\mathrm{H}(11 \mathrm{D})$ & 470 & 6654 & -588 & 57 \\
\hline $\mathrm{H}(115)$ & -141 & 7255 & 410 & 47 \\
\hline $\mathrm{H}(116)$ & 241 & 4453 & 926 & 45 \\
\hline $\mathrm{H}(11 \mathrm{E})$ & -365 & 4973 & 1968 & 55 \\
\hline $\mathrm{H}(11 \mathrm{~F})$ & -589 & 6537 & 1695 & 55 \\
\hline $\mathrm{H}(11 \mathrm{G})$ & -1784 & 5143 & 1542 & 55 \\
\hline $\mathrm{H}(11 \mathrm{H})$ & -1325 & 4000 & 1095 & 55 \\
\hline $\mathrm{H}(120)$ & -862 & 4619 & -32 & 48 \\
\hline $\mathrm{H}(12 \mathrm{~A})$ & -1012 & 5802 & -1080 & 62 \\
\hline $\mathrm{H}(12 \mathrm{~B})$ & -1274 & 7249 & -711 & 62 \\
\hline $\mathrm{H}(12 \mathrm{C})$ & -2296 & 4913 & -1082 & 68 \\
\hline $\mathrm{H}(12 \mathrm{D})$ & -2587 & 6477 & -886 & 68 \\
\hline $\mathrm{H}(123)$ & -2278 & 4128 & 34 & 56 \\
\hline $\mathrm{H}(12 \mathrm{C})$ & 556 & 7444 & 2081 & 78 \\
\hline
\end{tabular}




$\begin{array}{lrrrr}\mathrm{H}(12 \mathrm{D}) & 767 & 8158 & 1354 & 78 \\ \mathrm{H}(12 \mathrm{E}) & 1495 & 7759 & 1949 & 78 \\ \mathrm{H}(12 \mathrm{~F}) & -1988 & 7878 & 223 & 83 \\ \mathrm{H}(12 \mathrm{G}) & -1306 & 7834 & 890 & 83 \\ \mathrm{H}(12 \mathrm{H}) & -2246 & 7450 & 990 & 83 \\ \mathrm{H}(126) & -3373 & 6479 & 127 & 69 \\ \mathrm{H}(12 \mathrm{I}) & -3068 & 5526 & 1279 & 122 \\ \mathrm{H}(12 \mathrm{~J}) & -3350 & 3993 & 992 & 122 \\ \mathrm{H}(12 \mathrm{~K}) & -4013 & 5240 & 1002 & 122 \\ \mathrm{H}(12 \mathrm{I}) & -3653 & 4816 & -850 & 80 \\ \mathrm{H}(12 \mathrm{~J}) & -3816 & 3641 & -276 & 80 \\ \mathrm{H}(12 \mathrm{~L}) & -4787 & 6120 & -438 & 118 \\ \mathrm{H}(12 \mathrm{M}) & -4986 & 4743 & 4 & 118 \\ \mathrm{H}(13 \mathrm{~A}) & -5069 & 4963 & -1502 & 90 \\ \mathrm{H}(13 \mathrm{~B}) & -5096 & 3468 & -1120 & 90 \\ \mathrm{H}(13 \mathrm{~A}) & -4900 & 4468 & -1481 & 90 \\ \mathrm{H}(13 \mathrm{~B}) & -5391 & 3481 & -981 & 90 \\ \mathrm{H}(131) & -6371 & 3962 & -745 & 78 \\ \mathrm{H}(13 \mathrm{C}) & -7164 & 4079 & -1860 & 127 \\ \mathrm{H}(13 \mathrm{D}) & -6422 & 2973 & -1880 & 127 \\ \mathrm{H}(13 \mathrm{E}) & -6354 & 4505 & -2229 & 127 \\ \mathrm{H}(13 \mathrm{~F}) & -7030 & 6151 & -1057 & 139 \\ \mathrm{H}(13 \mathrm{G}) & -6221 & 6678 & -1398 & 139 \\ \mathrm{H}(13 \mathrm{H}) & -6193 & 6413 & -561 & 139 \\ \mathrm{H}(31 \mathrm{~B}) & -5910 & 6235 & -1474 & 101 \\ \mathrm{H}(32 \mathrm{~A}) & -6163 & 4430 & -2323 & 147 \\ \mathrm{H}(32 \mathrm{~B}) & -7023 & 4941 & -2065 & 147 \\ \mathrm{H}(32 \mathrm{C}) & -6612 & 3497 & -1768 & 147 \\ \mathrm{H}(33 \mathrm{~A}) & -6278 & 5906 & -298 & 218 \\ \mathrm{H}(33 \mathrm{~B}) & -6666 & 4391 & -504 & 218 \\ \mathrm{H}(33 \mathrm{C}) & -7100 & 5794 & -830 & 218 \\ \mathrm{H}(20 \mathrm{~A}) & -2433 & 6652 & 2983 & 92 \\ \mathrm{H}(20 \mathrm{~B}) & -1536 & 6650 & 2702 & 92 \\ \mathrm{H}(20 \mathrm{C}) & -1748 & 7755 & 3299 & 92 \\ \mathrm{H}(202) & -361 & 7539 & 3359 & 64 \\ \mathrm{H}(203) & -852 & 4166 & 4542 & 75\end{array}$




$\begin{array}{lrlll}\mathrm{H}(204) & 1008 & 7484 & 3846 & 62 \\ \mathrm{H}(205) & 522 & 4086 & 5009 & 70 \\ \mathrm{H}(207) & 2068 & 6691 & 4632 & 48 \\ \mathrm{H}(20 \mathrm{D}) & 2111 & 3663 & 4586 & 50 \\ \mathrm{H}(20 \mathrm{E}) & 2240 & 4655 & 3920 & 50 \\ \mathrm{H}(20 \mathrm{~F}) & 1649 & 6381 & 5800 & 51 \\ \mathrm{H}(20 \mathrm{G}) & 1771 & 4709 & 5726 & 51 \\ \mathrm{H}(21 \mathrm{~A}) & 2957 & 5674 & 6347 & 47 \\ \mathrm{H}(21 \mathrm{~B}) & 3056 & 6779 & 5723 & 47 \\ \mathrm{H}(213) & 3583 & 4677 & 3721 & 47 \\ \mathrm{H}(21 \mathrm{C}) & 4884 & 5762 & 4229 & 50 \\ \mathrm{H}(21 \mathrm{D}) & 4965 & 4103 & 4088 & 50 \\ \mathrm{H}(215) & 4981 & 3547 & 5258 & 42 \\ \mathrm{H}(216) & 4364 & 6324 & 5496 & 42 \\ \mathrm{H}(21 \mathrm{E}) & 4351 & 5816 & 6696 & 48 \\ \mathrm{H}(21 \mathrm{~F}) & 4682 & 4250 & 6585 & 48 \\ \mathrm{H}(21 \mathrm{G}) & 5784 & 5623 & 7082 & 47 \\ \mathrm{H}(21 \mathrm{H}) & 5625 & 6789 & 6472 & 47 \\ \mathrm{H}(220) & 5803 & 6201 & 5259 & 43 \\ \mathrm{H}(22 \mathrm{~A}) & 6502 & 5099 & 4445 & 52 \\ \mathrm{H}(22 \mathrm{~B}) & 6515 & 3608 & 4857 & 52 \\ \mathrm{H}(22 \mathrm{C}) & 7621 & 5844 & 5124 & 49 \\ \mathrm{H}(22 \mathrm{D}) & 7712 & 4274 & 5447 & 49 \\ \mathrm{H}(223) & 6994 & 6688 & 6038 & 43 \\ \mathrm{H}(22 \mathrm{C}) & 3738 & 2614 & 5591 & 67 \\ \mathrm{H}(22 \mathrm{D}) & 2797 & 2965 & 5702 & 67 \\ \mathrm{H}(22 \mathrm{E}) & 3512 & 3315 & 6317 & 67 \\ \mathrm{H}(22 \mathrm{~F}) & 6671 & 2955 & 6052 & 73 \\ \mathrm{H}(22 \mathrm{G}) & 5745 & 2958 & 6269 & 73 \\ \mathrm{H}(22 \mathrm{H}) & 6494 & 3357 & 6844 & 73 \\ \mathrm{H}(226) & 7861 & 4366 & 6731 & 49 \\ \mathrm{H}(22 \mathrm{I}) & 7901 & 5686 & 7795 & 95 \\ \mathrm{H}(22 \mathrm{~J}) & 6968 & 5313 & 7514 & 95 \\ \mathrm{H}(22 \mathrm{~K}) & 7321 & 6867 & 7402 & 95 \\ \mathrm{H}(22 \mathrm{I}) & 8705 & 5883 & 6102 & 55 \\ \mathrm{H}(22 \mathrm{~J}) & 8435 & 7200 & 6554 & 55\end{array}$




$\begin{array}{lrrrr}\mathrm{H}(22 \mathrm{~K}) & 9119 & 6236 & 7590 & 61 \\ \mathrm{H}(22 \mathrm{~L}) & 9357 & 4863 & 7162 & 61 \\ \mathrm{H}(23 \mathrm{~A}) & 9949 & 7579 & 6811 & 60 \\ \mathrm{H}(23 \mathrm{~B}) & 10289 & 6090 & 6567 & 60 \\ \mathrm{H}(231) & 10500 & 7164 & 7980 & 59 \\ \mathrm{H}(23 \mathrm{~A}) & 11372 & 5266 & 8287 & 106 \\ \mathrm{H}(23 \mathrm{~B}) & 11174 & 4604 & 7512 & 106 \\ \mathrm{H}(23 \mathrm{C}) & 10460 & 4722 & 8035 & 106 \\ \mathrm{H}(23 \mathrm{D}) & 11332 & 8357 & 7220 & 92 \\ \mathrm{H}(23 \mathrm{E}) & 11758 & 6893 & 7039 & 92 \\ \mathrm{H}(23 \mathrm{~F}) & 11884 & 7519 & 7827 & 92 \\ \end{array}$


Table 6. Torsion angles $\left[^{\circ}\right]$ for $04134 \mathrm{dpm}$.

\begin{tabular}{lc}
\hline$C(100)-O(1)-C(101)-C(103)$ & $177.0(3)$ \\
$C(100)-O(1)-C(101)-C(102)$ & $-3.5(4)$ \\
$O(1)-C(101)-C(102)-C(105)$ & $179.4(3)$ \\
$C(103)-C(101)-C(102)-C(105)$ & $-1.1(4)$ \\
$O(1)-C(101)-C(103)-C(104)$ & $-179.2(3)$ \\
$C(102)-C(101)-C(103)-C(104)$ & $1.2(4)$ \\
$C(101)-C(103)-C(104)-C(106)$ & $-0.6(5)$ \\
$C(101)-C(102)-C(105)-C(106)$ & $0.3(5)$ \\
$C(103)-C(104)-C(106)-C(105)$ & $-0.3(4)$ \\
$C(103)-C(104)-C(106)-C(107)$ & $175.2(3)$ \\
$C(102)-C(105)-C(106)-C(104)$ & $0.4(4)$ \\
$C(102)-C(105)-C(106)-C(107)$ & $-175.1(3)$ \\
$C(104)-C(106)-C(107)-C(109)$ & $44.6(4)$ \\
$C(105)-C(106)-C(107)-C(109)$ & $-140.1(3)$ \\
$C(104)-C(106)-C(107)-C(108)$ & $-78.8(4)$ \\
$C(105)-C(106)-C(107)-C(108)$ & $96.5(3)$ \\
$C(106)-C(107)-C(108)-C(112)$ & $-174.6(2)$ \\
$C(109)-C(107)-C(108)-C(112)$ & $58.7(3)$ \\
$C(106)-C(107)-C(109)-C(110)$ & $178.2(2)$ \\
$C(108)-C(107)-C(109)-C(110)$ & $-56.4(3)$ \\
$C(107)-C(109)-C(110)-C(113)$ & $-127.4(3)$ \\
$C(107)-C(109)-C(110)-C(111)$ & $52.2(3)$ \\
$C(113)-C(110)-C(111)-C(124)$ & $-106.8(3)$ \\
$C(109)-C(110)-C(111)-C(124)$ & $73.6(3)$ \\
$C(113)-C(110)-C(111)-C(116)$ & $15.3(4)$ \\
$C(109)-C(110)-C(111)-C(116)$ & $-164.3(2)$ \\
$C(113)-C(110)-C(111)-C(112)$ & $134.6(3)$ \\
$C(109)-C(110)-C(111)-C(112)$ & $-45.0(3)$ \\
$C(107)-C(108)-C(112)-C(111)$ & $-56.4(3)$ \\
$C(110)-C(111)-C(112)-C(108)$ & $47.0(3)$ \\
$C(124)-C(111)-C(112)-C(108)$ & $-71.1(3)$ \\
$C(116)-C(111)-C(112)-C(108)$ & $167.0(2)$ \\
$C(109)-C(110)-C(113)-C(114)$ & $-179.6(3)$ \\
$C(111)-C(110)-C(113)-C(114)$ & $0.8(5)$ \\
&
\end{tabular}




$\begin{array}{lc}C(110)-C(113)-C(114)-C(115) & 12.8(4) \\ C(113)-C(114)-C(115)-C(120) & -162.0(2) \\ C(113)-C(114)-C(115)-C(116) & -41.1(3) \\ C(120)-C(115)-C(116)-C(117) & -50.5(3) \\ C(114)-C(115)-C(116)-C(117) & -172.0(2) \\ C(120)-C(115)-C(116)-C(111) & -179.4(2) \\ C(114)-C(115)-C(116)-C(111) & 59.2(3) \\ C(110)-C(111)-C(116)-C(117) & -172.4(2) \\ C(124)-C(111)-C(116)-C(117) & -52.3(3) \\ C(112)-C(111)-C(116)-C(117) & 68.1(3) \\ C(110)-C(111)-C(116)-C(115) & -45.1(3) \\ C(124)-C(111)-C(116)-C(115) & 75.0(3) \\ C(112)-C(111)-C(116)-C(115) & -164.6(2) \\ C(115)-C(116)-C(117)-C(118) & 52.1(3) \\ C(111)-C(116)-C(117)-C(118) & -179.3(2) \\ C(116)-C(117)-C(118)-C(119) & -56.1(3) \\ C(117)-C(118)-C(119)-C(125) & -66.2(3) \\ C(117)-C(118)-C(119)-C(120) & 56.0(3) \\ C(117)-C(118)-C(119)-C(123) & 166.1(2) \\ C(114)-C(115)-C(120)-C(121) & -56.5(3) \\ C(116)-C(115)-C(120)-C(121) & -177.6(2) \\ C(114)-C(115)-C(120)-C(119) & 178.8(2) \\ C(116)-C(115)-C(120)-C(119) & 57.7(3) \\ C(125)-C(119)-C(120)-C(121) & -69.8(3) \\ C(118)-C(119)-C(120)-C(121) & 168.6(2) \\ C(123)-C(119)-C(120)-C(121) & 47.4(3) \\ C(125)-C(119)-C(120)-C(115) & 62.1(3) \\ C(118)-C(119)-C(120)-C(115) & -59.5(3) \\ C(123)-C(119)-C(120)-C(115) & 179.3(2) \\ C(115)-C(120)-C(121)-C(122) & -167.5(2) \\ C(119)-C(120)-C(121)-C(122) & -37.4(3) \\ C(120)-C(121)-C(122)-C(123) & 12.3(3) \\ C(121)-C(122)-C(123)-C(126) & \\ C(121)-C(122)-C(123)-C(119) & \\ C(125)-C(119)-C(123)-C(126) & \\ C(120)-C(119)-C(123)-C(126) & \\ & \\ C & \end{array}$




$\begin{array}{lc}C(118)-C(119)-C(123)-C(126) & 82.7(3) \\ C(125)-C(119)-C(123)-C(122) & 79.8(3) \\ C(120)-C(119)-C(123)-C(122) & -38.5(3) \\ C(118)-C(119)-C(123)-C(122) & -152.3(2) \\ C(122)-C(123)-C(126)-C(127) & -177.5(3) \\ C(119)-C(123)-C(126)-C(127) & -56.0(4) \\ C(122)-C(123)-C(126)-C(128) & 58.8(3) \\ C(119)-C(123)-C(126)-C(128) & -179.7(3) \\ C(127)-C(126)-C(128)-C(129) & 69.1(4) \\ C(123)-C(126)-C(128)-C(129) & -165.5(3) \\ C(126)-C(128)-C(129)-C(130) & 168.7(3) \\ C(128)-C(129)-C(130)-C(131) & 168.0(5) \\ C(128)-C(129)-C(130)-C(31 B) & -157.7(6) \\ C(31 B)-C(130)-C(131)-C(132) & 86.5(15) \\ C(129)-C(130)-C(131)-C(132) & 176.6(9) \\ C(31 B)-C(130)-C(131)-C(133) & -36.4(10) \\ C(129)-C(130)-C(131)-C(133) & 53.7(9) \\ C(131)-C(130)-C(31 B)-C(32 B) & -68.8(15) \\ C(129)-C(130)-C(31 B)-C(32 B) & -175.0(11) \\ C(131)-C(130)-C(31 B)-C(33 B) & 51.2(11) \\ C(129)-C(130)-C(31 B)-C(33 B) & -55.0(14) \\ C(200)-O(2)-C(201)-C(203) & -176.4(3) \\ C(200)-O(2)-C(201)-C(202) & 3.7(4) \\ C(203)-C(201)-C(202)-C(204) & -0.3(5) \\ \text { O(2)-C(201)-C(202)-C(204)} & 179.6(3) \\ C(202)-C(201)-C(203)-C(205) & -0.4(5) \\ \text { O(2)-C(201)-C(203)-C(205) } & 179.7(3) \\ C(201)-C(202)-C(204)-C(206) & 0.8(5) \\ C(201)-C(203)-C(205)-C(206) & 0.6(5) \\ C(202)-C(204)-C(206)-C(205) & -0.6(4) \\ C(202)-C(204)-C(206)-C(207) & 178.9(3) \\ C(203)-C(205)-C(206)-C(204) & -0.2(5) \\ C(203)-C(205)-C(206)-C(207) & -179.6(3) \\ C(204)-C(206)-C(207)-C(209) & -48.6(4) \\ C(205)-C(206)-C(207)-C(209) & \\ C(204)-C(206)-C(207)-C(208) & \\ & \end{array}$




$\begin{array}{lc}C(205)-C(206)-C(207)-C(208) & 75.0(3) \\ C(206)-C(207)-C(208)-C(210) & 174.1(2) \\ C(209)-C(207)-C(208)-C(210) & -58.7(3) \\ C(206)-C(207)-C(209)-C(212) & -173.7(2) \\ C(208)-C(207)-C(209)-C(212) & 59.9(3) \\ C(207)-C(208)-C(210)-C(213) & -126.5(3) \\ C(207)-C(208)-C(210)-C(211) & 52.9(3) \\ C(213)-C(210)-C(211)-C(224) & -105.5(3) \\ C(208)-C(210)-C(211)-C(224) & 75.1(3) \\ C(213)-C(210)-C(211)-C(212) & 135.6(3) \\ C(208)-C(210)-C(211)-C(212) & -43.8(3) \\ C(213)-C(210)-C(211)-C(216) & 16.3(3) \\ C(208)-C(210)-C(211)-C(216) & -163.1(2) \\ C(207)-C(209)-C(212)-C(211) & -55.9(3) \\ C(210)-C(211)-C(212)-C(209) & 45.4(3) \\ C(224)-C(211)-C(212)-C(209) & -73.4(3) \\ C(216)-C(211)-C(212)-C(209) & 165.0(2) \\ C(208)-C(210)-C(213)-C(214) & 179.8(2) \\ C(211)-C(210)-C(213)-C(214) & 0.4(4) \\ C(210)-C(213)-C(214)-C(215) & 11.5(4) \\ C(213)-C(214)-C(215)-C(220) & -161.3(2) \\ C(213)-C(214)-C(215)-C(216) & -39.4(3) \\ C(220)-C(215)-C(216)-C(217) & -50.1(3) \\ C(214)-C(215)-C(216)-C(217) & -172.6(2) \\ C(220)-C(215)-C(216)-C(211) & -179.2(2) \\ C(214)-C(215)-C(216)-C(211) & 58.4(3) \\ C(210)-C(211)-C(216)-C(215) & -45.3(3) \\ C(224)-C(211)-C(216)-C(215) & 74.9(3) \\ C(212)-C(211)-C(216)-C(215) & -164.9(2) \\ C(210)-C(211)-C(216)-C(217) & -173.0(2) \\ C(224)-C(211)-C(216)-C(217) & -52.7(3) \\ C(212)-C(211)-C(216)-C(217) & 67.4(3) \\ C(215)-C(216)-C(217)-C(218) & 50.9(3) \\ C(211)-C(216)-C(217)-C(218) & \\ C(216)-C(217)-C(218)-C(219) & \\ C(217)-C(218)-C(219)-C(225) & \\ & \\ C & \end{array}$




\begin{tabular}{lc}
$C(217)-C(218)-C(219)-C(220)$ & $54.9(3)$ \\
$C(217)-C(218)-C(219)-C(223)$ & $165.5(2)$ \\
$C(214)-C(215)-C(220)-C(221)$ & $-54.8(3)$ \\
$C(216)-C(215)-C(220)-C(221)$ & $-177.2(2)$ \\
$C(214)-C(215)-C(220)-C(219)$ & $180.0(2)$ \\
$C(216)-C(215)-C(220)-C(219)$ & $57.6(3)$ \\
$C(218)-C(219)-C(220)-C(215)$ & $-59.1(3)$ \\
$C(225)-C(219)-C(220)-C(215)$ & $62.8(3)$ \\
$C(223)-C(219)-C(220)-C(215)$ & $178.4(2)$ \\
$C(218)-C(219)-C(220)-C(221)$ & $168.5(2)$ \\
$C(225)-C(219)-C(220)-C(221)$ & $-69.7(3)$ \\
$C(223)-C(219)-C(220)-C(221)$ & $46.0(2)$ \\
$C(215)-C(220)-C(221)-C(222)$ & $-164.6(2)$ \\
$C(219)-C(220)-C(221)-C(222)$ & $-34.0(3)$ \\
$C(220)-C(221)-C(222)-C(223)$ & $8.3(3)$ \\
$C(221)-C(222)-C(223)-C(226)$ & $150.3(2)$ \\
$C(221)-C(222)-C(223)-C(219)$ & $20.1(3)$ \\
$C(218)-C(219)-C(223)-C(226)$ & $80.0(3)$ \\
$C(225)-C(219)-C(223)-C(226)$ & $-48.1(3)$ \\
$C(220)-C(219)-C(223)-C(226)$ & $-165.6(2)$ \\
$C(218)-C(219)-C(223)-C(222)$ & $-154.2(2)$ \\
$C(225)-C(219)-C(223)-C(222)$ & $77.8(2)$ \\
$C(220)-C(219)-C(223)-C(222)$ & $-39.8(2)$ \\
$C(222)-C(223)-C(226)-C(227)$ & $-176.3(2)$ \\
$C(219)-C(223)-C(226)-C(227)$ & $-54.7(3)$ \\
$C(222)-C(223)-C(226)-C(228)$ & $60.1(3)$ \\
$C(219)-C(223)-C(226)-C(228)$ & $-178.3(2)$ \\
$C(227)-C(226)-C(228)-C(229)$ & $60.1(3)$ \\
$C(223)-C(226)-C(228)-C(229)$ & $-175.1(2)$ \\
$C(226)-C(228)-C(229)-C(230)$ & $176.8(3)$ \\
$C(228)-C(229)-C(230)-C(231)$ & $166.1(3)$ \\
$C(229)-C(230)-C(231)-C(232)$ & $57.4(4)$ \\
$C(229)-C(230)-C(231)-C(233)$ & $-176.5(3)$ \\
& \\
\hline &
\end{tabular}

Symmetry transformations used to generate equivalent atoms: 


\section{References}

(1) Labadie, J. W.; Tueting, D.; Stille, J. K. J. Org. Chem. 1983, 48, 4634-4642.

(2) This substrate was prepared according to a literature procedure. See: Bajwa, J. S.; Anderson, R. C. Tetrahedron Lett. 1991, 32, 3021-3024.

(3) Kevill, D. N.; Weitl, F. L. J. Org. Chem. 1967, 32, 2633-2634.

(4) For a related procedure, see: Artis, D. R.; Cho, I.-S.; Jaime-Figueroa, S.; Muchowski, J. M. J. Org. Chem. 1994, 59, 2456-2466.

(5) Hackmann, C.; Schäfer, H. J. Tetrahedron 1993, 49, 4559-4574.

(6) (a) Grutzner, J. B.; Jautelat, M.; Dence, J. B.; Smith, R. A.; Roberts, J. D. J. Am. Chem. Soc. 1970, 92, 7107-7120. (b) Eda, M.; Takemoto, T.; Ono, S.-i.; Okada, T.; Kosaka, K.; Gohda, M.; Matzno, S.; Nakamura, N.; Fukaya, C. J. Med. Chem. 1994, 37, 19831990.

(7) Ferreira, J. T. B.; Cruz, W. O.; Vieira, P. C.; Yonashiro, M. J. Org. Chem. 1987, 52, 3698-3699.

(8) Pezechk, M.; Brunetiere, A. P.; Lallemand, J. Y. Tetrahedron Lett. 1986, 27, 3715-3718. 